\title{
Die Finanzierung direktdemokratischer Instrumente
}

\author{
Benjamin Karras
}

I. Finanzierung als Rechtsproblem

1. Marktwirtschaft und private Beteiligung am Staatswesen

a) Geld regiert die Welt

In marktwirtschaftlichen Systemen gewinnt die Frage der Finanzierung einer jeden Entscheidung eine besondere, teils beherrschende Bedeutung. Dies gilt auch und insbesondere für politische Entscheidungen. Nicht von ungefähr kreisen Wahlkampfdebatten und der parlamentarische Diskurs häufig um pekuniäre Differenzen, wie etwa das konfliktträchtige Immergrün Steuerrecht. Doch nicht nur der inhaltliche Disput ist auf das Engste mit dem Thema Geld verknüpft, auch die formalen Rahmenbedingungen zur Politikgestaltung selbst weisen weitreichende Interdependenzen auf. Das beginnt in den niedrigeren Politikebenen der Kommunalverwaltung, die noch von ehrenamtlichem Engagement geprägt sind, und zieht sich hoch bis zu Ministeriumskreisen, die für Gesetzesentwürfe private Kanzleien beauftragen. Angesichts der weiterhin anhaltenden Nachbeben der Finanzkrise 2008 und des daraus resultierenden Spardiktats bedarf es keiner prophetischen Gabe, die Fortdauer dieses bestimmenden Einflusses vorherzusagen.

b) „Wutbürger“ und Partizipation

Als weitere Tendenz der letzten Jahre zeichnet sich ein zunehmender Ruf nach direkter Bürgerpartizipation am Staatsgeschehen ab. Dies kulminierte bspw. in der „Stuttgart $21^{\text {“"-Debatte }}{ }^{1}$ und wurde ebenfalls in den Diskussio-

* Die Arbeit entstand im Rahmen eines Seminars zu aktuellen Rechtsfragen der direkten Demokratie bei Prof. Dr. Fabian Wittreck. Der Verfasser dankt Prof. Wittreck herzlich für seine außerordentliche Unterstützung und wohlwollenden Ratschläge. 
nen um eine Direktwahl des Bundespräsidenten offenkundig ${ }^{2}$. Größere Aufmerksamkeit erregten gleichfalls einige bedeutsame Volksgesetzgebungsverfahren, etwa zum Rauchverbot in Bayern ${ }^{3}$ oder zum Verbot des Minarettbaus in der Schweiz ${ }^{4}$. Neu entfacht wurde zugleich der Streit um die Einführung plebiszitärer Elemente auf Bundesebene ${ }^{5}$. Doch auch bestehende Möglichkeiten werden erneut und verschärft unter das prüfende Auge der Wissenschaft gezogen. Ansatzpunkt der Kritik ist die vergleichsweise marginale Relevanz unmittelbarer Demokratie im staatlichen Gefüge der Bundesrepublik Deutschland ${ }^{6}$. Einigkeit innerhalb der Literatur findet die Ansicht, dass die Verfahrensregelungen maßgebenden Einfluss auf die Häufigkeit und Erfolgsaussichten direktdemokratischer Instrumente ausüben ${ }^{7}$. Eine Aufwertung

1 Dazu nur O. Jung, Stuttgart 21 und die Direkte Demokratie, in: RuP 48 (2012), S. 11 ff.

2 D. Grimm, Der Bundespräsident, FAZ v. 19.1.2012 Nr. 16, S. 19; Günter Bannas, Zwischen Verfassungstheorie und Verfassungswirklichkeit, FAZ v. 19.3.2012 Nr. 67, S. 3; Interview mit Rita Süssmuth: „Das Volk soll direkt wählen“, SZ-online am 29.6.2010, verfügbar unter http://www.sueddeutsche.de/politik/wahl-desbundes-praesidenten-rita-suessmuth-ich-werbe-fuer-die-direktwahl-des-bundespraesidenten-1.966542, zuletzt abgerufen am 15.2.2014.

$3 H$. Schoen/A. Glantz/R. Teusch, Abstimmungskampf, Informationsvermittlung und Stimmentscheidung beim Volksentscheid über den Nichtraucherschutz in Bayern, in: L.P. Feld/P.M. Huber/O. Jung/C. Welzel/F. Wittreck (Hrsg.), Jahrbuch für direkte Demokratie 2010, 2011, S. $295 \mathrm{ff}$.

4 Hierzu A. Tschentscher/D. Blonski, Direkte Demokratie in der Schweiz - Länderbericht 2010/2011, in: Feld u.a. Jahrbuch 2010 (Fn. 3), S. 169 (171 ff.); H.K. Heußner, Minarettverbot in der Schweiz: Argument gegen Volksentscheide in Deutschland? Zur Diskussion über Volksgesetzgebung, Minderheitenschutz und Völkerrecht -, in: RuP 46 (2010), S. 19 ff.

5 Dazu in aller Breite F. Decker, Das Volk als Gesetzgeber? Zur Diskussion um die Einführung plebiszitärer Elemente auf Bundesebene, in: ders. (Hrsg.), Regieren im „Parteienbundesstaat“. Zur Architektur der deutschen Politik, 2011, S. 165 ff.; siehe auch $H$. Dreier/F. Wittreck, Repräsentative und direkte Demokratie im Grundgesetz, in: L.P. Feld/P.M. Huber/O. Jung/C. Welzel/F. Wittreck (Hrsg.), Jahrbuch für direkte Demokratie 2009, 2010, S. $11 \mathrm{ff}$.

$6 \mathrm{Zu}$ diesem Fazit gelangt etwa J. Rux, Direkte Demokratie in Deutschland, 2002, S. 904 ff.; markant S. Przygode, Die deutsche Rechtsprechung zur unmittelbaren Demokratie. Ein Beitrag zur Praxis der Sachentscheide in Deutschland, 1995, S. 38: „Schattendasein“.

7 Dazu nur O. Jung, Verfahrensprobleme der Volksgesetzgebung, in: ZG 8 (1993), S. 314 (315 f.); H. Abromeit, Nutzen und Risiken direktdemokratischer Instrumente, in: C. Offe (Hrsg.), Demokratisierung der Demokratie. Diagnosen und Reformvorschläge, 2003, S. 95 (110); Przygode, Rechtsprechung (Fn. 6), S. 42; Rux, Demokratie (Fn. 5), S. 106 f. Vgl. auch die sehr detaillierten Vorschläge in O. Jung, Welche Regeln empfehlen sich bei der Einführung von Volksbegehren und Volksentscheid 
des Plebiszits soll demnach vor allem durch eine partizipationsfreundlichere Ausgestaltung der Abstimmungsmodalitäten erfolgen.

\section{c) Schnittstelle}

Die wahrgenommenen Tendenzen verdichten sich bei der Frage der Kostenerstattung von Abstimmungskämpfen. Formal sind die direktdemokratischen Verfahren nicht an finanzielle Mindestleistungen gebunden, jedoch lassen sich in praxi ähnliche Marketingmechanismen wie für den Wahlkampf beobachten. Oftmals werden private Initiatoren sammelnd oder werbend tätig, was naturgemäß mit Unkosten verbunden ist. Der betriebene Werbeaufwand variiert von Kampagne zu Kampagne, konstituiert aber in jedem Fall eine nicht unbeachtliche finanzielle Belastung. Dies gilt umso mehr, als die plebiszitären Verfahren gerade den ,gewöhnlichen“ Bürgern zur Verfügung stehen sollen, deren institutionelle und ökonomische Absicherung in dieser demokratischen Willensbetätigung nicht ausschlaggebendes Kriterium für einen Erfolg sein darf.

Das Thema der Kostenerstattung für direktdemokratische Instrumente spielte in der rechtswissenschaftlichen Diskussion in Deutschland jedoch bisher eine untergeordnete Rolle ${ }^{8}$ und systematische Untersuchungen dazu finden sich fast ausschließlich in der Schweiz ${ }^{9}$ und besonders in den USA. Noch 1993 konnte Otmar Jung festhalten: „Das ganze Thema Abstimmungskampfkosten-Erstattung ist neu, jede Regelung innovativ und noch nirgendwo praktische Erfahrung vorhanden“ " ${ }^{10}$. Angesichts der immer noch geringen Relevanz im Verhältnis zu den Parlamentswahlen erschöpft sich die

(Volksgesetzgebung) auf Bundesebene?, in: Evangelische Akademie Hofmeisgar/Stiftung MITARBEIT (Hrsg.), Direkte Demokratie in Deutschland. Handreichungen zur Verfassungsdiskussion in Bund und Ländern. Mit Entwürfen zur Einführung von Volksbegehren und Volksentscheid auf Bundesebene (,Hofmeisgarer Entwurf"), 1991, S. 19 (21 ff.), die veranschaulichen, welcher Stellenwert dem Verfahrensrecht eingeräumt wird.

8 Diese Tatsache bemängelt auch Przygode, Rechtsprechung (Fn. 6), S. 453.

9 Gleichwohl spricht M. Caroni, Herausforderung Demokratie, in: ZSR 132 (2013), S. 5 (79) davon, dass diese Fragestellung auch in der Schweiz, ,weitgehend vernachlässigt" worden ist.

10 Jung, Verfahrensprobleme (Fn. 7), S. 335. 
Diskussion auch heute noch zumeist in rechtspolitischen Forderungen, ohne einen verfassungsrechtlichen Bezug herzustellen ${ }^{11}$.

\section{Finanzierung als Rechtsproblem}

In der Bundesrepublik Deutschland wird ausgehend von Art. 3 Abs. 1 GG die Gleichheit aller Bürger als Verfassungsgrundsatz behandelt. Schon der Wortlaut, also „gleich vor dem Gesetz“, zeigt, dass es sich dabei um eine rechtliche Gleichheit handelt. Nicht hingegen ist die faktische Gleichheit, die Gleichheit aller tatsächlichen Lebensumstände, umfasst ${ }^{12}$. Denn notwendigerweise ergibt sich aus den folgenden Freiheitsverbürgungen, die der Einzelne wahrnehmen kann, aber eben nicht muss, dass je nach Gebrauch unterschiedliche Grade der Verwirklichung erreicht werden. Konsequenz dieser vorgefundenen Divergenzen ist demnach eine je nach wirtschaftlicher Potenz unterschiedliche faktische Entfaltungsmöglichkeit, die, gleichsam grundgesetzlich abgesegnet, hinzunehmen ist. Dieses ohnehin liberalindividualistisch gedachte Grundrechtsverständnis ${ }^{13}$ lässt sich aber nicht in gleicher Weise auf die Partizipationschancen des einzelnen Bürgers übertragen, geht doch der demokratisch verfasste Staat des Grundgesetzes in seiner Gemeinwohlorientierung ${ }^{14}$ von der Relevanz eines jeden Individuums aus ${ }^{15}$.

11 So etwa bei O. Jung, Grundsatzfragen der direkten Demokratie, in: A. Kost (Hrsg.), Direkte Demokratie in den deutschen Ländern, 2005, S. 312 (332 f.); oder auch bei dems., Regeln (Fn. 7), S. 51 ff., der sich dort freilich im Rahmen der Diskussion eines Gesetzgebungsvorschlages äußert; etwas ausführlicher, aber gleichwohl nur mit losem Verfassungsbezug T. Schiller, Finanzierungsregeln: Offenlegungspflichten, Spendenbegrenzungen, Kostenerstattung, in: T. Mörschel/M. Efler (Hrsg.), Direkte Demokratie auf Bundesebene, 2013, S. 131 (140 ff.).

$12 \mathrm{Zu}$ diesem verfassungsrechtlichen Allgemeinplatz siehe nur C. Starck, Grundrechtliche und demokratische Freiheitsidee, in: J. Isensee/P. Kirchhof (Hrsg.), HStR III, 3. Aufl. 2005, § 33 Rn. 25.

13 Weitergehende soziale Grundrechtsvoraussetzungen betont etwa J. Isensee, Grundrechtsvoraussetzungen und Verfassungserwartungen, in: ders./P. Kirchhof (Hrsg.), HStR IX, 3. Aufl. $2011 \S 190$ Rn. 184 ff.; die ausgleichende Integrationsfunktion des Sozialstaatsprinzips legt Starck (Fn. 12), § 33 Rn. 26, dar. Darüber hinaus ignoriert eine solche Konzeption die staatliche Schutzpflicht, einen effektiven Grundrechtsgebrauch zu ermöglichen; dazu nur BVerfGE 7, 230 (233 f.); 25, 256 (263); 73, 261 (269); 93, 1 (16); 114, 1 (37 f.).

14 Siehe hierzu etwa ausführlich $M$. Anderheiden, Gemeinwohl in Republik und Union, 2006; die verfassungsrechtlichen und verfassungsrichterlichen Aspekte dieses schillernden Begriffs beleuchten H. Hofmann, Verfassungsrechtliche Annäherungen an den Begriff des Gemeinwohls, in: H. Münkler/K. Fischer (Hrsg.), Gemeinwohl und 
Damit werden bereits das partizipationsbeeinflussende Vorfeld und die Durchführung des Verfahrens selbst Betrachtungsgegenstand des Verfassungsrechts. Hinzu kommt, dass die Verfahrens- und Durchführungsmodalitäten rechtlich normiert werden und diese Gesetze verfassungskonform gefasst sein müssen. Ein rechtsfreier, dem ungebundenen Spiel bestehender Ungleichheiten überlassener Partizipationsraum kann somit unter der Ägide des Grundgesetzes nicht bestehen. Damit müssen aber gleichfalls die praktisch sehr bedeutsamen ${ }^{16}$ Finanzierungsregelungen in ihrer Ausgestaltung kritisch durchleuchtet werden. Die Finanzierung direktdemokratischer Instrumente kann daher nicht einfachhin als rechtlich unbeachtlich abgetan werden.

\section{Gegenstand und Gang der Untersuchung}

Diese Untersuchung soll den aktuellen Rechtsstand zur Frage der Kostenerstattung in Deutschland aufbereiten (II.) und in das Norm- und Struktursystem der Bundesrepublik einordnen. Dazu sind die Vorgaben des Grundgesetzes (III.) und Folgerungen aus dem parallel gelagerten Rechtsinstitut der Wahlkampfinanzierung (IV.) zu ermitteln. Sodann sind die Kostenbedürfnisse der direktdemokratischen Instrumente und deren Einfluss auf die Entscheidung zu untersuchen (V.), bevor die gegenwärtige Rechtslage daran gemessen werden kann (VI.). Dergestalt entwickelte Maßstäbe sind - unter Berücksichtigung rechtsvergleichender Hinweise - in einem rechtspolitischen Entwurf praxisgerecht umzusetzen und zur Debatte zu stellen (VII.). Dabei sind unter direktdemokratischen Instrumenten die Volksinitiative, das Volksbegehren und der Volksentscheid in der Ausgestaltung des jeweiligen Landesrechts zu verstehen, ohne speziell Bezug auf die kommunalen Instrumente zu nehmen.

Angesichts des begrenzten Umfangs dieses Beitrags muss eine inhaltliche Einschränkung vorgenommen werden. Der Schwerpunkt der Darstellung liegt auf der Frage der öffentlichen Finanzierung direktdemokratischer In-

Gemeinsinn im Recht, 2002, S. 25 ff. und D. Grimm, Gemeinwohl in der Rechtsprechung des Bundesverfassungsgerichts, ebda., S. 125 ff. Weitere Ausführungen zum Verhältnis der Bürger zur Gemeinwohlorientierung finden sich bei J. Isensee, Gemeinwohl im Verfassungsstaat, in: ders./P. Kirchhof (Hrsg.) HStR IV, 3. Aufl. 2006, $\S 71 \mathrm{Rn} .110 \mathrm{ff}$.

15 Siehe unter III.2.

16 Hierzu unter V.2. 
strumente. Denkbar wäre anderweitig die Reglementierung privater Zuwendung in Gestalt einer Beschränkung der Höhe nach oder von Offenlegungspflichten ${ }^{17}$. Gänzlich unberücksichtigt muss vorliegend die Möglichkeit einer Spendenbeschränkung bleiben. Dies ergibt sich aus zwei Gesichtspunkten: Zum Ersten gibt es dazu keine Regelungen in Deutschland, und auch für den Wahlkampf ist dies nicht vorgesehen. Zweitens spielt diese Variante in der rechtswissenschaftlichen Diskussion keine Rolle. Das eine überschneidet sich mit dem anderen, wenn man sich die verfassungsrechtlichen Probleme vor Augen führt, die mit einer solchen Einschränkung einhergehen ${ }^{18}$. Deren Evidenz kann im gegebenen Rahmen nicht hinterfragt werden. Offenlegungspflichten als Korrelat zu einer öffentlichen Finanzierung hingegen können kurz beim abschließenden Gesetzgebungsvorschlag berührt werden.

\section{Gegenwärtige (Nicht-)Regelungen}

Von den 16 Bundesländern haben bisher erst acht Länder gesetzliche Regelungen zur Kostenerstattung von direkt-demokratischen Instrumenten erlassen. Dies sind Baden-Württemberg, Hamburg, Niedersachen, Sachsen, Sachsen-Anhalt, Schleswig-Holstein und Thüringen. Für die Bundesebene stand mangels praktischen Anwendungsfalles ein solches Regelwerk nie zur Debatte. Das einzig allgemein anerkannte plebiszitäre Element des Bundesrechts, der Volksentscheid zur Neugliederung des Bundesgebietes nach Art. 29 Abs. 2 S. $1 \mathrm{GG}^{19}$, hat noch keine Ausweitung zu einem durchnormierten Rechtsinstitut mit Verfahrensbestimmung und eben Kostenregelungen erfahren.

17 Siehe zu diesen beiden Aspekten bezogen auf die Bundesrepublik - meines Wissens erstmalig - Schiller, Finanzierungsregeln (Fn. 11), S. 133 ff. bzw. 136 ff.

18 Parteien wurzeln nach der Rechtsprechung im gesellschaftlichen Bereich und sollen ihre Willensbildung (dazu gehört auch die Finanzierung) staatsfrei vollziehen; BVerfGE 20, 56 (101 ff.); 73, 40 (97); 85264 (287 ff.). Hinzu kommen grundrechtliche Aspekte für die Spender wie die Meinungsfreiheit, Vereinigungsfreiheit für Mitglieder. Wegen der Möglichkeit von z.B. Offenlegungspflichten oder gestaffelter steuerlicher Absetzbarkeit dürfte allein der Verhältnismäßigkeitsgrundsatz kaum zu wahren sein.

19 Die Anerkennung als Plebiszit ist unabhängig davon, ob man die Territorialplebiszite des Grundgesetzes (Art. 29 GG, sowie dessen historische Anwendungsfälle in Art. 118, 118a GG) als Abstimmungen im Sinne des Art. 20 Abs. 2 S. 2 GG ansieht; ablehnend etwa H. Dreier, in: ders. (Hrsg.), Grundgesetz. Kommentar, Bd. 2, 2. Aufl. 2006, Art. 20 (Demokratie), Rn. 104, mit weiteren Nachweisen. 


\section{Baden-Württemberg}

Denkbar gering fällt die Kostenerstattung in Baden-Württemberg aus. Nach $\S 39$ Abs. 2 VAbstG BW ${ }^{20}$ werden den Antragssteller Kosten nur erstattet, wenn die Volksabstimmung zur Auflösung des Landtags führt. Allerdings sind dann nur die Kosten des Zulassungsantrags sowie die Kosten der Eintragungslisten und ihrer Versendung an die Gemeinden zu ersetzen.

\section{Hamburg}

In Hamburg behandelt $\S 30 \mathrm{a}$ des Volksabstimmungsgesetzes ${ }^{21}$ (HmbVAbstG) die Frage der Kostenerstattung. Dabei können den Initiatoren eines Volksentscheides für jede Ja-Stimme bis zu $0,10 €$ ersetzt werden. Für andere direktdemokratische Verfahren sind die Akteure auf private Mittel angewiesen. Diese Erstattung im Falle eines Volksentscheides ist auf 400.000 JaStimmen begrenzt und kann damit bestenfalls $40.000 €$ betragen. Im Ergebnis werden maximal ein Drittel der u.U. abgegebenen Stimmen der 1,2 Mio. Hamburger Wahlberechtigten ${ }^{22}$ den Initiatoren finanzwirksam zugeschrieben. Versäumen es die Initiatoren, den nach $\S 30 \mathrm{HmbVAbstG}$ erforderlichen Rechenschaftsbericht über die Verwendung der Mittel vorzulegen, so verlieren sie nach $\S 30$ Abs. 3 HmbVAbstG jeden Rechtsanspruch auf Kampagnenfinanzierung.

\section{Niedersachsen}

Die niedersächsische Verfassung gebietet in Art. 50 Abs. 1 jedenfalls für ein zustande gekommenes Volksbegehren die „Erstattung der notwendigen Kosten einer angemessenen Information der Öffentlichkeit über die Ziele des Volksbegehrens“. In Abs. 2 findet sich ein Gesetzgebungsauftrag zur Regelung der Kostenerstattung für Volksinitiative, Volksbegehren und Volksent-

20 Gesetz über Volksabstimmung und Volksbegehren vom 27. Februar 1984.

21 Hamburgisches Gesetz über Volksinitiative, Volksbegehren und Volksentscheid vom 20. Juni 1996.

22 Pressemittelung des Statistischen Bundesamtes zur voraussetzungsidentischen Bundestagswahl 2009, verfügbar unter http://www.bundeswahlleiter.de/de/bundestagswahlen/BTW_BUND_09/presse/11Wahlberechtigte_Laender.html, abgerufen am 7.2.2014. 
scheid, der mit dem Volksabstimmungsgesetz (NdsVAbstG) erfüllt wurde. $\S 39$ Abs. 1 NdsVAbstG wiederholt lediglich Art. 50 Abs. 1 NdsV, § 39 Abs. 2 und 3 NdsVAbstG treffen Regelungen über eine Kostenerstattung zugunsten der Gemeinden und Gemeindeverbände. In Niedersachen kann somit nur im Falle eines zustande gekommenen Volksbegehrens Ersatz der Kampagnenkosten verlangt werden. Der Betrag wird dabei durch $\S 6$ Abs. 1 NdsWahlKostVO ${ }^{23}$ auf $0,10 €$ je gültige und für das Zustandekommen notwendige Stimme pauschaliert. Darüber hinausgehende Kosten können nach $\S 6$ Abs. 2 NdsWahlKostVO auf Antrag ersetzt werden, sofern sie notwendig zur Information der Öffentlichkeit sind. Über diesen Antrag entscheidet gemäß $§ 8$ NdsWahlKostVO das für Wahlen zuständige Landesministerium, also das Innenministerium.

\section{Rheinland-Pfalz}

An sich ist die Kostenerstattung in Rheinland-Pfalz für jegliches direktdemokratische Verfahren ausgeschlossen. Ausnahmen hierzu finden sich einzig in $\S 76$ Abs. 3 LWahlG RP ${ }^{24}$ : Dort werden zum einen die Kosten der Antragsteller erstattet, die im Rahmen der Herstellung von Eintragungslisten und ihrer Versendung an die Gemeindeverwaltungen entstanden sind - die Kostentragungspflicht ergibt sich aus $\S 76$ Abs. 1 LWahlG RP. Allerdings gilt dies nur bei Annahme durch das Parlament oder bei einem erfolgreichen anschließenden Volksentscheid. Die weitere Ausnahme betrifft die Landtagsauflösung, die durch Volksentscheid erreicht wird; auch hier wird der Erfolg vorausgesetzt. Rheinland-Pfalz kennt eine Kostenerstattung daher nur im engsten Sinne, nämlich dass Kosten, die zwangsläufig bei der Durchführung eines direktdemokratischen Verfahrens bei Behörden anfallen, nicht mehr den Antragstellern auferlegt werden.

23 Verordnung über die Erstattung von Kosten bei Landtags- und bei Kommunalwahlen sowie im Zusammenhang mit Volksbegehren und Volksentscheiden vom 26. Mai 2009.

24 Landeswahlgesetz vom 24. November 2004. 


\section{Sachsen}

Das Land Sachsen hat die Kostenfrage für jedes direktdemokratische Instrument isoliert geregelt. $\S 15$ Abs. 1 SächsVVVG ${ }^{25}$ bestimmt, dass für die Volkinitiative die Antragsteller die Kosten bis zur Einreichung beim Landtagspräsidenten selbst zu tragen haben. Dies gilt nach $\S 6$ Abs. 3 Satz 1 SächsVVVG nicht für die Bestätigung der Unterschriftengültigkeit durch die Gemeinde. Für das Volksbegehren schreibt § 24 Abs. 1 SächsVVVG vor, dass den Antragstellern grundsätzlich alle notwendigen Organisationskosten ersetzt werden, ohne dass es auf den erfolgreichen Ausgang des Begehrens ankäme. In Abs. 2 wird dieser Betrag auf $0,51 €$ je zehn gültige Unterschriften festgesetzt. Die Zahl der berücksichtigungsfähigen Stimmberechtigten wird auf 450.000 begrenzt. Für den Volksentscheid werden die erstattungsfähigen Kosten auf 1,02 $€$ pro 100 gültiger Ja-Stimmen pauschalisiert, § 47 Abs. 2 SächsVVVG. Auch hier ist der Ausgang des Volksentscheides für die Frage der Kostenerstattung unerheblich.

\section{Sachsen-Anhalt}

Sachsen-Anhalt erstattet in $\S 31$ Abs. 1 VAbstG LSA ${ }^{26}$ für ein angenommenes Volksbegehren einen Pauschalbetrag von $0,26 €$ pro erforderlicher Unterschrift. In $\S 31$ Abs. 2 VAbstG LSA wird derselbe Betrag je Ja-Stimme für den Volksentscheid festgeschrieben. Allerdings sind nur 25\% der Stimmen der Beteiligungsberechtigten erstattungsfähig, darüber hinausgehende JaStimmen werden nicht berücksichtigt. Jedoch ist es für die Erstattung bezüglich eines Volksentscheides nicht erheblich, ob er erfolgreich war. Die Erstattungssumme darf gemäß $\S 31$ Abs. 3 VAbstG LSA nachgewiesenermaßen nicht über den Betrag der Werbungsmaßnahmen hinausgehen.

25 Gesetz über Volksantrag, Volksbegehren und Volksentscheid vom 19. Oktober 1993. Zu diesem Gesetz vgl. V. Krieg, Die Volksgesetzgebung im Freistaat Sachsen, in: ZG 11 (1996), S. 314 ff.

26 Gesetz über das Verfahren bei Volksinitiative, Volksbegehren und Volksentscheid (Volksabstimmungsgesetz - VAbstG) vom 26. Oktober 2005. 


\section{Schleswig-Holstein}

In Schleswig-Holstein bestimmt Art. 42 Abs. 3 Satz 2 LV SH, dass den Antragstellern die notwendigen Kosten der angemessenen Werbung jedenfalls für den Volksentscheid erstattet werden müssen. Darauf beschränkt sich auch die einfachgesetzliche Ausgestaltung durch das Volksabstimmungsgesetz $\left(\right.$ VAbstG SH) ${ }^{27}$. 27 Abs. 2 Satz 1 VAbstG SH gewährt $0,28 €$ je gültiger Ja-Stimme, ohne dass nach Abs. 2 Satz 2 VAbstG SH die erstatteten Kosten die tatsächlich nachgewiesenen übersteigen dürfen.

\section{Thüringen}

In Thüringen wiederum wird zwischen Bürgerantrag, Volksbegehren und Volksentscheid differenziert. Die Kosten für die Herstellung der Unterschriftsbögen und deren Übermittlung an die kreisfreien Städte und Landkreise sind gemäß $§ 29$ Abs. 1 Satz 1 ThürBVVG ${ }^{28}$ von den Antragstellern zu tragen. Die notwendigen und nachgewiesenen Organisationskosten für ein zustande gekommenes Volksbegehren werden den Antragstellern mit 0,15€ je gültiger Unterschrift ersetzt, allerdings nur, soweit diese für das Zustandekommen erforderlich sind, § 29 Abs. 2 Satz 2, Satz 3 ThürBVVG. Auch die Kostenerstattung für den Volkentscheid ist von einem erfolgreichen Ausgang abhängig. Nach § 29 Abs. 3 Satz 2 können den Antragstellern pro gültiger Ja-Stimme 0,075 $€$ erstattet werden. Die Anzahl berücksichtigungsfähiger Stimmen ist gleichfalls auf die zum Erfolg notwendigen Stimmen begrenzt. Der Antrag auf Kostenerstattung ist für das Volksbegehren nach § 29 Abs. 2 Satz 4 ThürBVVG, für den Volksentscheid nach Abs. 3 Satz 4 schriftlich beim Landtagspräsidenten zu stellen.

27 Gesetz über Initiativen aus dem Volk, Volksbegehren und Volksentscheid in der Fassung vom 5. April 2004.

28 Thüringer Gesetz über das Verfahren bei Bürgerantrag, Volksbegehren und Volksentscheid in der Fassung der Neubekanntmachung vom 23. Februar 2004 (GVB1. S. 237). 


\section{Der gemeinsame Nenner der Konzepte}

Vergleicht man die in den Bundesländern gegenwärtig vorhanden Regelungskonzepte $^{29}$, so lassen sich diese kategorial unterscheiden, obwohl kein Modell detailgleich in zwei Ländern auftaucht. Dies betrifft zum einen die direktdemokratischen Instrumente, für die überhaupt eine Kostenerstattung vorgesehen ist, und zum anderen die Frage, ob dieses Instrument im Sinne der Antragsteller erfolgreich gewesen sein muss.

a) Kostenerstattung nach Verfahrensart

Nicht ein Bundesland ermöglicht eine Kostenerstattung für die Volksinitiative. Für das Volksbegehren sind staatliche Mittel in Niedersachen, Sachsen, Sachsen-Anhalt und Thüringen zu erhalten. Die Organisatoren eines Volksentscheides können in allen Ländern mit Ausnahme von Niedersachsen finanzielle Unterstützung durch das Land beantragen. Schleswig-Holstein und Hamburg sehen damit im Volksentscheid das direktdemokratische Instrument, das am ehesten der Finanzierung bedarf, wohingegen Sachsen-Anhalt, Sachsen und Thüringen zumindest bei der Frage des „Ob“ einer Kostenerstattung nicht zwischen Volksentscheid und Volksbegehren unterscheiden. Eine paradoxe Rechtslage herrscht in Niedersachsen: Dort wird der Werbeaufwand für ein Volksbegehren für unterstützungswürdig erachtet, wohingegen eine Finanzierung des Volksentscheides nicht stattfindet.

\section{b) Erfolgsabhängigkeit}

Die zweite Unterscheidungsmöglichkeit betrifft die Erfolgsabhängigkeit der Finanzierung. Ein Volksbegehren muss in Niedersachsen, Sachsen-Anhalt und Thüringen erfolgreich sein, damit die Initiatoren eine Kostenerstattung beantragen können. Für den Volksentscheid ist dagegen nur in Thüringen allgemein die Annahme zu verlangen.

29 Was im Bereich der unmittelbaren Demokratie mitunter schwierig sein kann, für Rux, Demokratie (Fn. 6), S. 904, ist ,in keinem anderen Bereich der Föderalismus so lebendig, wie hier“. Gleichwohl erkennt auch er „wesentliche Grundstrukturen“ an. 
c) Sonderwege in Baden-Württemberg und Rheinland-Pfalz

Sonderwege beschreiten hingegen Baden-Württemberg und Rheinland-Pfalz. Wegen der aufgezeigten Einschränkungen hinsichtlich des Gegenstandes eines als finanzierungswürdig angesehenen direktdemokratischen Verfahrens kann hier von allgemeiner Kostenerstattung von vornherein keine Rede sein. Erschwerend kommt hinzu, dass in diesen beiden Bundesländern lediglich die Kosten der bürokratischen Verfahrensdurchführung vom Staat getragen werden. Eine Kos-tenerstattung im engeren Sinne, also eine Abgeltung des Aufwandes des Abstimmungskampfes, findet somit gar nicht statt. Es wäre daher sachlich gerechtfertigt, auch in diesen Ländern das Fehlen eines Regelungswerkes zur hier zu untersuchenden Thematik zu konstatieren.

\section{Die normativen Leitlinien des Demokratieprinzips}

Den Ausgangspunkt einer verfassungsrechtlichen Erörterung ${ }^{30}$ der Finanzierungsproblematik muss Art. 20 Abs. 1 und 2 GG bilden. Dabei finden sich in Abs. 1 das grundsätzliche Bekenntnis des Grundgesetzes zur Demokratie und in Abs. 2 der Begründungsansatz (Volkssouveränität) sowie eine weitere Ausdifferenzierung im Hinblick auf die Ausübung der Staatsgewalt ${ }^{31}$. Das Bekenntnis des Grundgesetzes zur Demokratie ist für den staatsrechtlichen Aufbau der Bundesrepublik Deutschland fundamental. In der früheren Rechtsprechung und Literatur diente Art. 20 GG vor allem dazu, Staatshandlungen hinsichtlich ihrer Legitimität an den Vorgaben des Abs. 2 Satz $1 \mathrm{zu}$ $\operatorname{messen}^{32}$. Von beträchtlichem Gewicht sind aber gleichfalls die verfahrens-

30 Aufschluss- und einsichtsreich für die Verfassungsverhältnisse der Schweiz ist die neuere Behandlung der Problematik bei Caroni, Herausforderung (Fn. 9), S. 60 ff. Dort werden die verfassungsrechtlichen Determinanten maßgeblich anhand der politischen bzw. Abstimmungsgleichheit sowie (nachrangig) der Vereinigungsfreiheit entwickelt.

31 Ebenso E.-W. Böckenförde, Demokratie als Verfassungsprinzip, in: J. Isensee/P. Kirchhof (Hrsg.), HStR II, 3. Aufl. 2004, § 24 Rn. 8; B. Grzeszick, in: T. Maunz/G. Dürig (Begr.), Grundgesetz. Kommentar, Art. 20 II (2010), Rn. 11 f.; U. Volkmann, in: K.H. Friauf/W. Höfling (Hrsg.), Berliner Kommentar zum Grundgesetz, Art. 20 C (2001), Rn. 44.

32 Vgl. die grundlegenden Entscheidungen zur „Legitimationskette“ BVerfGE 47, 253 (275); 107, 59 (87). In neuerer Zeit wurde dies im Lissabon-Urteil im Begriff des „Legitimationsniveaus“ fruchtbar gemacht, BVerfGE 123, 267 (364 ff.). 
rechtlichen Dimensionen des Art. 20 Abs. 1 und 2 GG $^{33}$. Sind für das Wahlverfahren rechtliche Garantien zum Teil in Art. 38 Abs. 1 GG expressis verbis enthalten, so schweigt sich das Grundgesetz zur Frage der Abstimmung in Gänze aus ${ }^{34}$. Verfassungsrechtliche Anforderungen an die Verfahrensregelungen der Länder (und hypothetisch auch des Bundes) sind also unmittelbar aus Art. 20 Abs. 1 und 2 GG zu gewinnen. Sind dabei Verfahrensregelungen in die Landesverfassung selbst integriert worden, wie es etwa in Niedersachsen und Schleswig-Holstein der Fall ist, so sind auch diese Bestimmungen wegen des Homogenitätsgebotes des Art. 28 Abs. 1 Satz 1 GG an den demokratiebezogenen Kernvorgaben des Grundgesetzes zu messen ${ }^{35}$.

\section{Demokratie als Staatsprinzip}

Mit der Aufzählung in Art. 20 Abs. 1 S. 1 GG verbürgt das Grundgesetz den demokratischen und sozialen Bundesstaat. Die Norm statuiert zu Beginn des staatsorganisationsrechtlichen Abschnittes des Grundgesetzes solche Grundentscheidungen, die wegen der Ewigkeitsgarantie des Art. 79 Abs. 3 GG zumindest im Rahmen der gegenwärtigen Verfassung unabänderlich sind ${ }^{36}$.

Im vorliegenden Zusammenhang ist vor allem die Wirkungsweise des Demokratieprinzips von Belang. Rechtsdogmatisch betrachtet ist das Demokratieprinzip kein normativer Rechtssatz, der Tatbestand und Rechtsfolge anordnet $^{37}$. Vielmehr dient diese organisationsrechtliche Determinante als Prinzipienbestimmung. Prinzipien sind nun aber auf Verwirklichung angelegt, und zwar durch graduelle Annäherung an den typisierten Idealzustand ${ }^{38}$.

33 Aus der Rechtsprechung siehe BVerfGE 71, 81 (94); 123, 267 (341 ff.).

34 Außer Betracht bleibt der Volksentscheid zur Neugliederung des Bundesgebiets nach Art. 29 Abs. 2 S. 1 GG, der eine sehr spezielle und daher nicht verallgemeinerungsfähige Form eines Plebiszits regelt.

35 Zum Homogenitätsgebot im Allgemeinen vgl. nur J. Kersten, Homogenitätsgebot und Landesverfassungsrecht, in: DÖV 1993, S. 896 (897 ff.); den Bezug zur direkten Demokratie stellt $P$. Krause, Verfassungsrechtliche Möglichkeiten unmittelbarer Demokratie, in: Isensee/Kirchhof, HStR III (Fn. 12), § 35 Rn. 27, her.

36 Die problematische Frage der Geltung im Falle einer Ablösung nach Art. 146 GG bleibt hier außer Betracht.

37 So zutreffend F.E. Schnapp, in: I. v. Münch/P. Kunig (Hrsg.), GG Kommentar, Bd. 1, 6. Aufl. 2012, Art. 20 Rn. 6; zu den unterschiedlichen rechtsdogmatischen Zugriffen siehe ausführlich Volkmann (Fn. 31), Art. 20 C Rn. 46 ff.

38 Grundlegend zu der kategorialen Unterscheidung von Regel und Prinzip R. Alexy, Theorie der Grundrechte, 1985, S. 75 ff., der von „Optimierungsgeboten“ spricht. An diese Terminologie schließen sich auch inhaltlich an Schnapp (Fn. 37), Art. 20 
Ausgangspunkt für die materielle Festlegung dieses Verfassungsziels sind nicht rechts- oder verfassungspolitische Wünsche des Normanwenders ${ }^{39}$, sondern ist der durch die regulären Methoden zu ermittelnde normative Gehalt des Demokratieprinzips ${ }^{40}$. Obwohl der Begriff der Demokratie in Art. 20 GG abstrakt und unbestimmt bleibt, ist demnach eine Annäherung über konkretisierende Normen möglich ${ }^{41}$. Allen Konzepten, so divers sie in ihrer Gestalt und in ihren Forderungen auch sein mögen, sind gewisse Grundbestimmungen gemeinsam ${ }^{42}$, auf die es im Folgenden ankommt. Als Mindestkriterien $^{43}$ zur Charakterisierung dieser Herrschaftsform ${ }^{44}$ lässt sich die Voraussetzung politischer Selbstbestimmung von gleichberechtigten Bürgern ${ }^{45}$ und die Entscheidungsfindung nach dem Mehrheitsprinzip ${ }^{46}$ festhalten, die nicht

Rn. 7; B.-O. Bryde, Das Demokratieprinzip des Grundgesetzes als Optimierungsaufgabe, in: Redaktion Kritische Justiz (Hrsg.), Demokratie und Grundgesetz, 2000, S. 59 (62 ff.).

39 Diese Unterscheidung bekräftigt B. Pieroth, in: H.D. Jarass/ders., GG, 12. Aufl. 2012, Art. 20 Rn. 1.

40 Näher M. Jestaedt, Demokratieprinzip und Kondominialverwaltung, 1993, S. 145 f.

41 Zum Doppelcharakter als Rechtsbegriff und politischer Utopie - was die juristische Präzisierung erschwert - siehe Dreier (Fn. 19), Art. 20 (Demokratie), Rn. 66. Die Kennzeichnung als „Idealbegriff“ findet sich bereits bei C. Schmitt, Verfassungslehre, 1. Aufl. 1928, S. 225. Das Verhältnis von Demokratiebegriff und entsprechenden Konkretisierungen im Grundgesetz beleuchten Schnapp (Fn. 37), Art. 20 Rn. 4; K. Hesse, Grundzüge des Verfassungsrechts der Bundesrepublik Deutschland, 20. Aufl. 1995, Rn. 127 und Pieroth (Fn. 39), Art. 20 Rn. 1. Zu den vielschattigen Konzeptionen von Demokratie vgl. nur Jestaedt, Demokratieprinzip (Fn. 40), S. 138 ff.

42 So auch Schnapp (Fn. 37), Art. 20 Rn. 18.

43 Umfassende Versuche zur Bestimmung dieser Mindestkriterien unternehmen Grzeszick (Fn. 31), Art. 20 II, Rn. 10 ff.; Dreier (Fn. 19), Art. 20 (Demokratie), Rn. 73 ff.; M. Sachs, in: ders. (Hrsg.), Grundgesetz Kommentar, 6. Aufl. 2011, Art. 20 Rn. 16 ff.

44 Die Vorstellung von Demokratie als herrschaftslose Staatsorganisation darf als widerlegt angesehen werden, vgl. die Debatte zwischen $R$. Spaemann und J. Habermas, insb. R. Spaemann, Die Utopie der Herrschaftsfreiheit (1972), in: ders. (Hrsg.), Zur Kritik der politischen Utopie, 1977, S. 104 ff., mit den weiteren Beiträgen dort. Nicht Herrschaftsaufhebung, sondern eine spezifische Form der Herrschaftsorganisation macht die Demokratie aus: Böckenförde (Fn. 31), § 24 Rn. 9; Dreier (Fn. 19), Art. 20 (Demokratie), Rn. 68.

45 Vgl. nur die entsprechenden Passagen in BVerfGE 27, 71 (81); 44, 125 (139); 73, 40 (85).

46 BVerfGE 29, 154 (165); 70, 366 (368 f.); siehe dazu Sachs (Fn. 43), Art. 20 Rn. 21 ff. und die entsprechenden Verweise. 
auf unbegrenzte Zeit durch (in erster Linie) Wahlen ${ }^{47}$ an dazu berufene Stellen delegiert werden $\mathrm{kann}^{48}$.

Durch das Demokratieprinzip wird nicht nur dieser Inhalt, sondern zugleich auch die Art und Weise seiner Verwirklichung vorgegeben ${ }^{49}$. In seiner Bipolarität als Staatsziel und Strukturnorm wirkt sich das Demokratieprinzip nun sowohl auf die Modalitäten eines vorgesehenen demokratischen Verfahrens als auch auf dessen inhaltliche Legitimität aus. Gerade hierin zeigt sich Art und Ausmaß der Anforderungen, die Art. 20 Abs. 1 GG - insbesondere an den Gesetzgeber stellt. Schließlich ist das Demokratieprinzip zwar abstrakt $^{50}$, in seinem objektiv-rechtlichen Gehalt deshalb aber nicht weniger verbindlich. Der Gesetzgeber ist demnach dazu berufen, die rechtlichen Voraussetzungen dafür zu schaffen, dass den materiellen Forderungen Genüge getan wird. Dieser „Regelungssauftrag an den einfachen Gesetzgeber“51 nimmt für das Demokratieprinzip, ebenso wie für andere Staatszielbestimmungen, die Form eines Förder- und Schutzauftrages an ${ }^{52}$, der umfassend, also für alle Etappen des demokratischen Prozesses greift ${ }^{53}$. Für das Demokratieprinzip gilt dies in verstärktem Maße, beruht diese Herrschaftsform doch auf der Überzeugung, dass Verfahrensgerechtigkeit die Plausibilität der Erwartungshaltung auf Ergebnisgerechtigkeit verbürgt ${ }^{54}$. Es liegt also im Wesen des Demokratieprinzips, dass sich dessen rechtlicher Gehalt zu gro-

47 BVerfGE 18, 151 (154); 20, 56 (113 f.); 62, 1 (79); aus der Literatur vgl. nur Dreier (Fn. 19), Art. 20 (Demokratie), Rn. 79, mit weiteren Nachweisen.

$48 \mathrm{Zu}$ diesen Kriterien vgl. in der Gesamtschau K.-P. Sommermann, in: H. v. Mangoldt/F. Klein/C. Starck (Hrsg.), GG, Bd. II, 6. Aufl. 2010, Art. 20 Abs. 1 Rn. 81 ff.; Sachs (Fn. 43), Art. 20 Rn. 12; Dreier (Fn. 19), Art. 20 (Demokratie), Rn. 73 ff.; Schnapp (Fn. 37), Art. 20 Rn. 20 ff.

49 In diesem Sinne Volkmann (Fn. 31), C Art. 20 Rn. 36 ff.; A. v. Komorowski, Demokratieprinzip und Europäische Union, 2010, S. 587 f. Lediglich ein „Staatsstrukturprinzip“ erkennt Sommermann (Fn. 48), Art. 20 Abs. 1 Rn. 88 ff. an. Dabei verkennt Sommermann aber, dass bereits die Entscheidung für die Demokratie als Staatsform auf dem unterstellten Wertgehalt dieser Ordnung beruht und nicht nur auf prozeduraler Zweckmäßigkeit; wie hier Volkmann (Fn. 31), C Art. 20 Rn. 33.

50 Von einer ,auf höchster Abstraktionsstufe angesiedelte[r]“" Norm spricht v. Komorowski, Demokratieprinzip (Fn. 49), S. 586.

51 Volkmann (Fn. 31), C Art. 20 Rn. 40.

52 Volkmann (Fn. 31), C Art. 20 Rn. 40; ausführlich zur Gleichheitsaufgabe des Staates A. Nußberger, Soziale Gleichheit - Voraussetzung oder Aufgabe des Staates?, in: DVB1. 2008, S. 1081 (1087 f.).

53 Bereits das Vorfeld von Wahlen ist mit Anforderungen an demokratische Gleichheit durchdrungen, BVerfGE 8, 51 (61); 52, 63 (82 ff.); 73, 40 (71 ff.).

54 D. Merten, Bürgerverantwortung im demokratischen Verfassungsstaat, in: VVDStRL 55 (1995), S. 7 (23); Dreier (Fn. 19), Art. 20 (Demokratie), Rn. 84. 
ßen Teilen in Anforderungen an die Ausgestaltung des - gleichfalls vorausgesetzten - demokratischen Verfahrens niederschlägt ${ }^{55}$.

Wichtige Schlussfolgerung der Beschreibung von Demokratie als Staatsprinzip ist also die Feststellung, dass die realen Gegebenheiten und Prozesse demokratischer Willensbildung nicht stoisch hinzunehmen, sondern in Anlehnung an das grundgesetzliche Idealbild der Demokratie staatlicherseits zu gestalten sind.

\section{Demokratie als Gleichheitsverbürgung}

Im demokratischen Rechtsstaat wird die Janusköpfigkeit der Gleichheit offenkundig. Zunächst ist die Gleichheit der Staatsbürger Voraussetzung und auch Legitimationsquelle ${ }^{56}$ eines Gemeinwesens, dessen Verfassung den Einzelnen in die Mitte der staatlichen Letztbegründung stellt ${ }^{57}$. Andererseits ist diese Gleichheit nicht bloß apriorisches Postulat, sondern muss fortwährend errungen, beschützt oder wiederhergestellt werden. Die vom Demokratieprinzip vorausgesetzte und simultan eingeforderte Gleichheit ist also weder eine leere, unter Umständen tautologische, Begriffsformel noch eine unabweisbar festgeschriebene Normierung, sondern sie ist auf fortwirkende Verwirklichung angelegt ${ }^{58}$. Wenn Volkssouveränität und damit demokratische Herrschaft als unverzichtbarer Bestandteil der Verfassungsordnung nach Art. 79 Abs. 3 GG anerkannt wird ${ }^{59}$, dann muss dieser verfassungs-

55 Böckenförde (Fn. 31), § 24 Rn. 40.

56 So Starck (Fn. 12), §33 Rn. 32. Zum Zusammenhang von Legitimität und Gleichheit siehe weiter G. Jochum, Materielle Anforderungen an das Entscheidungsverfahren in der Demokratie, 1997, S. 59 f.; K. Herrmann, Volksgesetzgebungsverfahren, 2003, S. $41 \mathrm{f}$.

57 BVerfGE 123, 267 (341); C. Starck, in: H. von Mangoldt/F. Klein/ders. (Hrsg.), GG, Bd. I, 6. Aufl. 2010, Art. 1 Abs. 1 Rn. 10; Herrmann, Volksgesetzgebungsverfahren (Fn. 56), S. 39 ff.; S. Huster/J. Rux, in: V. Epping/C. Hillgruber (Hrsg.), GG, 2. Aufl. 2013, Art. 20, Vor Rn. 1; Dreier (Fn. 19), Art. 20 (Demokratie), Rn. 67, m.w.N.

$58 \mathrm{Zu}$ diesem Konzept Volkmann (Fn. 31), C Art. 20 Rn. 36. Aus dem Blickpunkt der sozialen Gleichheit gelangt Nußberger, Gleichheit (Fn. 52), S. 1086 ff. zu demselben Ergebnis.

59 BVerfGE 89, 155 (182); zur Gleichheit als Bedingung dieser Volksherrschaft auch M. Kriele, Das demokratische Prinzip im Grundgesetz, in: VVDStRL 29 (1970), S. 46 (61 ff.); H. Dreier, in: ders., GGK II (Fn. 19), Art. 79 Abs. 3 Rn. 38; K. Stern/M. Sachs, Das Staatsrecht der Bundesrepublik Deutschland, Band III/2, 1994, S. 1121. 
rechtlichen Forderung durch ein solches Wahl- und Verfahrensrecht Folge geleistet werden, das sich an dem grundlegenden Strukturprinzip ebendieser Demokratie orientiert, und dies ist die Gleichheit der Herrschaftssubjekte. Gleichheit der Herrschaftssubjekte wird in diesem Zusammenhang nicht als bloß rechtliche, sondern ebenso als wirtschaftliche Gleichheit verstanden, jedenfalls insoweit diese wirtschaftliche Gleichheit über Ein- oder Ausschluss in die politische Mitwirkung entscheidet ${ }^{60}$. Wichtiger Bestandteil der Gleichheitsgarantie ist somit nicht nur die strikte Gleichheit des Wahlrechts ${ }^{61}$, sondern insbesondere auch die Chancengleichheit im Wahlwettbewerb $^{62}$.

Demnach ist dem Demokratieprinzip jedenfalls dann nicht mehr Genüge getan, wenn faktische Ungleichheiten, beispielsweise auch finanzieller Natur, einen Kommunikationsprozess ${ }^{63}$ generell vereiteln. Hier ist auch ein Mindestmaß an demokratischer Mitwirkung nicht mehr gegeben. Zur rechtlichen Problematik erhebt sich dies allerdings nicht in erster Linie aus den vom Grundgesetz verliehenen Rechtspositionen, sondern aus dem Demokratieprinzip selbst. Ein im Mehrheitsprinzip erlassenes Gesetz kann nur dann Geltung gegenüber jedermann einfordern, wenn auch jedermann die Möglichkeit hatte, auf die Entstehung dieses Gesetzes einzuwirken ${ }^{64}$. Die Legitimität der Mehrheitsentscheidung fließt damit aus der Gleichheit der politischen Mitwirkungsrechte ${ }^{65}$. Nur ,wenn die Mehrheit aus einem freien, offenen, regelmäßig zu erneuernden Meinungs- und Willensbildungsprozess, an dem grundsätzlich alle wahlmündigen Bürger zu gleichen Rechten teilhaben

60 Diesen Aspekt betont Nußberger, Gleichheit (Fn. 52), S. 1086 f. im Rückgriff auf Hermann Heller.

61 Vgl. zu dieser verfassungsgerichtlichen Intensivierung des Gleichheitsmaßstabes nur BVerfGE 20, 56 (116); 34, 81 (99); 41, 399 (413); 78, 350 (357 f.); 85, 264 (297); 95, 408 (417); 104, 14 (19 f.).

62 Vgl. dazu etwa BVerfGE 24, 300 (339 ff.); 69, 92 (106 f.); 78, 350 (358); 85, 264 (297); dazu auch Sachs (Fn. 43), Art. 20 Rn. 19 f., der diesen Grundsatz ebenfalls nicht allein auf Parteien beschränkt.

63 Die Bedeutung des Diskurses hebt besonders hervor Jochum, Anforderungen (Fn. 56), S. 67 ff.

64 M. Reichel, Das demokratische Offenheitsprinzip und seine Anwendung im Recht der politischen Parteien, 1996, S. 109 f.; zur Bedeutung der tatsächlichen Gleichheit für das Mehrheitsprinzip auch Grzeszick (Fn. 31), Art. 20 II Rn. 46 ff.; Jochum, Anforderungen (Fn. 56), S. $47 \mathrm{ff}$.

65 Böckenförde (Fn. 31), § 24 Rn. 52; Grzeszick (Fn. 31), Art. 20 II Rn. 42 f.; Volkmann (Fn. 31), C. Art. 20 Rn. 26. 
können, hervorgegangen ist“66, kann diese Entscheidung Verbindlichkeit für alle Herrschaftsunterworfenen reklamieren. Staatlicherseits müssen daher Vorkehrungen zur institutionellen und faktischen Gewährleistung einer solchen Kommunikationsplattform getroffen werden. Dies gilt namentlich auch für faktische Verzerrungen im politischen und gesellschaftlichen Wettbewerb, denen entgegenzuwirken der Gesetzgeber qua Verfassung gehalten ist.

\section{Demokratie und Grundrechte ${ }^{67}$}

Schon die Kennzeichnung eines Staatswesens als Demokratie erfordert denknotwendig ein Mindestmaß an Gleichheit in den politischen Einwirkungsmöglichkeiten, um den Anspruch einer Mehrheitsentscheidung tragen zu können ${ }^{68}$. Damit ist zweierlei gesagt: zum einen müssen solche Einwirkungsmöglichkeiten überhaupt bestehen und zum anderen muss diese Form der Partizipation allen Staatsbürgern gleichermaßen offen stehen. Für den Wahlakt, die wichtigste Form demokratischer Teilhabe unter dem Grundgesetz, finden diese objektiv-rechtlichen Prinzipien ihre subjektiv-rechtliche Wendung vor allem in Art. 38 Abs. 1 S. 1 GG $^{69}$. Es wird nun zu zeigen sein, dass es für die rechtliche Bewertung direktdemokratischer Verfahren nicht, zumindest nicht primär, auf die subjektiven Rechte der Akteure ankommt, sondern diese subjektiv-rechtlichen Garantien Ausdruck der objektiven Strukturprinzipien sind, die ein umfassenderes, aber um nichts weniger verbindliches Rahmengerüst bieten ${ }^{70}$.

66 BVerfGE 44, 125 (142); die Bedeutung der Möglichkeit, eigene Auffassungen in dem demokratischen Prozess einzubringen, unterstreichen Huster/Rux (Fn. 57), Art. 20 Rn. 49 ff.

67 Dazu W. Schmitt Glaeser, Private Gewalt im politischen Meinungskampf, 1990, S. $21 \mathrm{ff}$.

68 F. Meyer, Wahlgrundsätze, Wahlverfahren, Wahlprüfung, in: Isensee/Kirchhof, HStR III (Fn. 12), § 46 Rn. 30.

69 P.M. Huber, Parteien in der Demokratie, in: P. Badura/H. Dreier (Hrsg.), Festschrift 50 Jahre Bundesverfassungsgericht, Bd. 2, 2001, S. 609 (610), sieht Art. 20 Abs. 2 S. 1 GG ,durch Art. 38 Abs. 1 GG subjektiv-rechtlich angereichert [...]?““.

70 Für den Bereich der Parteienfinanzierung hält Huber, Parteien (Fn. 69), S. 615, fest, „daß es des Rückgriffs auf die Grundrechte insoweit gar nicht bedarf“. 
Die in Art. 38 Abs. 1 S. 1 GG zugesagte Gleichheit ist in der Judikatur des Bundesverfassungsgerichts als strenge und formale Gleichheit aufgefasst worden $^{71}$. Das Bundesverfassungsgericht geht damit über die Maßstäbe des allgemeinen Gleichheitssatzes des Art. 3 Abs. 1 GG hinaus ${ }^{72}$ und sieht das Hauptargument im engen funktionalen Zusammenhang zum Demokratieprinzip des Art. 20 Abs. $1 \mathrm{GG}^{73}$. Art. 38 Abs. 1 Satz 1 GG statuiert ausweislich des eindeutigen Wortlauts lediglich die Wahlgrundsätze für die Wahl zum deutschen Bundestag. Der insoweit eingeschränkte Regelungsbereich kann aber zugunsten einer umfassenden Geltung auch für direktdemokratische Verfahren in den Ländern ausgeweitet werden, wenn die genannten Grundsätze zumindest in Teilen unmittelbar aus dem Demokratieprinzip des Art. 20 Abs. $1 \mathrm{GG}$ folgen. Dann bedürfte es keiner weiteren normativen Stütze mehr, um Grundsätze des Verfahrensrechts auch für die Abstimmungsmechanismen der Länder zu entwickeln. Entsprechend sieht das Bundesverfassungsgericht den strikten (subjektiv-rechtlich ausgestalteten) Gleichheitssatz des Art. 38 Abs. 1 S. 1 GG darin begründet, dass die „durch das Grundgesetz errichtete demokratische Ordnung [...] unbeschadet der bestehenden sozialen Unterschiede im Bereich der politischen Willensbildung alle Staatsbürger grundsätzlich gleich bewertet.“74 Wie eng der systematische Zusammenhang zwischen Wahl und Abstimmung ist, zeigt weiter die analoge Anwendung der Wahlrechtsgrundsätze auf Abstimmungen im allgemeinen durch das Bundesverfassungsgericht ${ }^{75}$. Diese Entscheidungen hatten zwar in der Sache jeweils Art. 29 GG zum Gegenstand, allerdings spricht das Bundesverfassungsgericht von ungeschriebenem Verfassungsrecht, das auch für sonstige politische Abstimmungen und Volksentscheide gilt ${ }^{76}$. Verbindendes Element von Wahl und Abstimmung, das diese Analogie begründet, ist wiederum die sozusagen demokratieakzessorische Ausübung von Staatsgewalt.

71 Grundlegend BVerfGE 11, 351 (360); als ständige Rechtsprechung weitergeführt in BVerfGE 20, 56 (116); 28, 220 (225); 34, 81 (99); 41, 399 (413); 51, 222 (234); 78, 350 (357 f.); 85, 264 (297); 95, 408 (417); 104, 14 (19 f.).

72 Dazu z.B. BVerfGE 78, 350 (357).

73 Etwa BVerfGE 82, 322 (337); 95, 408 (417).

74 So BVerfGE 51, 222 (234).

75 Vgl. BVerfGE 13, 54 (91 f.); 28, 220 (224); 29, 15 (19).

76 So etwa BVerfGE 41, 1 (12); dort stand eine gesetzliche Regelung zur Wahl einer Richtervertretung zur Debatte; für alle Abstimmungen unterstützt dies Sachs (Fn. 43), Art. 20 Rn. 34 
Dies bedeutet keine „Überfrachtung“ des Demokratieprinzips mit einfachgesetzlichen Ausführungsregelungen, sondern trägt dem umfassenden Verwirklichungsgebot Rechnung, das in der prägnanten Kurzformel des Art. 20 Abs. 1 komprimiert ist ${ }^{77}$. Diesem Ansatz entspricht ebenfalls die Tatsache, dass in Art. 28 Abs. 1 Satz 2 GG die Grundsätze des Art. 38 Abs. 1 Satz 1 GG für die Wahlen zu den Kommunal- und Landesvertretungen wiederholt werden. Die dadurch vorgenommene Wertung verdeutlicht die konstitutive Funktion des Wahlverfahrens, wie sie dem Demokratiebegriff des Grundgesetzes inhärent ist ${ }^{78}$. Die im Wahl- und Abstimmungsprozess einschlägige Gleichheitsidee fließt also im Wesentlichen aus dem Demokratieprinzip.

\section{b) Freiheit}

Im Rahmen politischer Willensbildung sind es hauptsächlich die allgemeinen Freiheitsgarantien der Grundrechte, die die Partizipationschancen des Bürgers rechtlich absichern. Diese Mitwirkungsrechte sind etwa die Kommunikationsrechte aus Art. 5 Abs. 1, Art. 8 und 9 GG, sowie, als Abwehrrecht im weiteren Sinne, die Parteienfreiheit aus Art. 21 Abs. 1 GG. Der verfassungsrechtliche Nexus zum Demokratieprinzip wird in der notwendigen Kommunikation und Erörterung im Rahmen der Demokratie hergestellt. Ebenso wie für die zeitliche und inhaltliche Ausdehnung der demokratischen Gleichheit in Gestalt der Chancengleichheit auf die entscheidungserheblichen Vorhandlungen ${ }^{79}$ lässt sich für die Demokratie selbst festhalten, dass sie sich als Herrschaftsorganisation nicht in einem einmaligen Wahlakt erschöpft, sondern auch konkrete strukturelle Vorbedingungen einfordert. So verstanden, folgt aus dem Demokratieprinzip unmittelbar, dass im Vorlauf einer Entscheidung die Vor- und Nachteile erörtert werden müssen. Ebenso muss den Kandidaten und Parteien im Wahlverfahren und den unterschiedlichen Interessenvertretern im Abstimmungsverfahren die Möglichkeit gegeben werden, sich selbst und ihre Anliegen öffentlichkeitswirksam zu präsentieren. Dies aber erfordert nun wiederum eine Mindestgewährleistung an kommunikati-

77 A. Tschentscher, Demokratische Legitimation der Dritten Gewalt, 2006, S. 11, spricht vom ,überschießenden Gehalt“ des Demokratieprinzips.

78 So auch Starck (Fn. 12), § 33 Rn. 32.; zur Bedeutung der Verfahrensbindung vgl. Jochum, Anforderungen (Fn. 56), S. 64 f.

79 Geschehen in BVerfGE 52, 63 (89 ff.); 73, 40 (89); 82, 322 (337 f.). Die enge Verknüpfung von Chancengleichheit und Wahlgleichheit wird unterstrichen in BVerfGE 20, 300 (340 ff.); 52, 63 (89). 
ver Freiheit, die eine diskursive Abwägung überhaupt erst ermöglicht. Das Bundesverfassungsgericht betont in ständiger Rechtsprechung, dass die kommunikativen Grundrechte des Art. 5 Abs. 1 GG „für eine freiheitlichdemokratische Staatsordnung [...] schlechthin konstituierend“" 80 sind. Es handelt sich dabei folglich erneut um die subjektiv-rechtliche Emanation der objektiven Geltung des Demokratieprinzips.

c) Schlussfolgerungen für die Finanzierung direktdemokratischer Instrumente

Aus diesen Ausführungen ergeben sich auch Rückschlüsse für die Finanzierung direktdemokratischer Instrumente. In diesen Abstimmungsprozessen sind, gerade im Hinblick auf wirtschaftliche Zusammenhänge, vielfach private Interessen involviert. Im grundrechtlich durchdrungenen Verfassungsstaat der Bundesrepublik Deutschland kann sich der Einzelne zur Durchsetzung und zum Schutz dieser Interessen im Regelfall auf eine Rechtsgarantie verlassen. Für die unmittelbaren demokratischen Verfahren ist aber bereits die antithetische Gegenüberstellung von Staat und Individuum verlassen, da sich die Partizipation gerade auf die Bildung des Staatswillens richtet. Demzufolge sind die Strukturprinzipien des Grundgesetzes, wie sie für die Staatsorganisation in Art. 20 GG niedergelegt sind, von vorrangiger, wenn auch nicht ausschließlicher Bedeutung ${ }^{81}$. Dies gilt trotz des „,subjektiv-rechtlichen Einschlages" auch für die private Partizipation. Im Ergebnis kann es daher für die Frage der Kostenerstattung nicht auf das Bestehen eines originären Leistungsanspruchs aus Grundrechten ${ }^{82}$ ankommen, vielmehr sind die allgemeinen Anforderungen des demokratischen Prinzips anzulegen.

Aus diesen Gründen kann vorliegend auch die Frage außer Acht gelassen werden, ob überhaupt eine Grundrechtsberechtigung der Beteiligten im Ab-

80 BVerfGE 7, 198 (208); bezugnehmend und weiter ausgeführt in BVerfGE 62, 230 (247); 76, 196 (208 f.); 102, 347 (363).

81 Noch deutlicher Huber, Parteien (Fn. 69), S. 623, der den Rückgriff auf Grundrechte als „,bestenfalls funktionslos“ einordnet; Stern/Sachs, Staatsrecht III/2 (Fn. 59), S. 1119 ff. hingegen betonen die grundrechtliche Relevanz des Art. 20 GG.

$82 \mathrm{Zu}$ dieser Problematik und dem eng begrenzten Anwendungsbereich siehe nur $E$. Denninger, Staatliche Hilfe zur Grundrechtsausübung, in: J. Isensee/P. Kirchhof (Hrsg.), HStR IX, 3. Aufl. 2011, § 193 Rn. 82 ff.; W. Rüfner, Leistungsrecht, in: D. Merten/J.-J. Papier (Hrsg.), HGR II, 2006, § 40 Rn. 11 ff. 
stimmungskampf existiert und wieweit diese gegebenenfalls reicht ${ }^{83}$. Zum rechtlichen Problem erwächst diese Unterscheidung nämlich erst in der Durchsetzung der Rechtsnormen. Unabhängig davon, ob diese nun subjektivrechtlich geltend gemacht werden können oder nicht, besteht doch gleichermaßen die objektiv-rechtliche Norm. Wenn - wie dies vorliegend getan wird - das Demokratieprinzip als weiterreichende Quelle objektiven Verfassungsrechts angesehen wird, wird die Frage nach der Grundrechtsberechtigung somit obsolet.

\section{Demokratie und Staatsgewalt}

Anknüpfungsobjekt für das Demokratieprinzip ist die Ausübung von Staatsgewalt. Im Ausgangspunkt ist daher diese tatbestandliche Voraussetzung Grenze des Anwendungsbereichs des Demokratieprinzips ${ }^{84}$. Die „Staatsgewalt" wird im Grundgesetz nicht näher ausgeführt, obwohl es sich um einen Kernbegriff des Staatsrechts handelt. Daher verbleibt es der Normauslegung, dieses zentrale Merkmal der Staatlichkeit mit Leben zu füllen. Im Anschluss an Jestaedt lässt sich zwischen materieller - bezogen auf die inhaltliche Bedeutung - und formeller - bezogen auf die Modalitäten - Staatsgewalt unterscheiden $^{85}$. Materiell liegt demnach Staatsgewalt vor, wenn eine Staatsaufgabe wahrgenommen wird. Von einer Staatsaufgabe wiederum lässt sich im Verfassungsstaat nur sprechen, wenn eine solche durch die Verfassung oder in verfassungskonformer Weise durch den Gesetzgeber bestimmt ist ${ }^{86}$. Unter

83 Eine Grundrechtsberechtigung lehnte das Bundesverfassungsgericht in BVerfGE 96, 231 (239 ff.) für den Abstimmungskampf im Rahmen eines Volksbegehrens ab. Dagegen wendet sich mit beachtlichen Argumenten B.J. Hartmann, Volksgesetzgebung und Grundrechte, 2005, S. 115 ff., der auf die Unterschiede zwischen Abgeordneten und Stimmbürgern rekurriert; in diese Richtung auch Herrmann, Volksgesetzgebungsverfahren (Fn. 56), S. 108.

84 Richtigerweise zurückhaltend gegenüber einer Ausweitung auf den rein gesellschaftlichen Bereich etwa E.-W. Böckenförde, Die Bedeutung der Unterscheidung von Staat und Gesellschaft im demokratischen Sozialstaat der Gegenwart, in: ders., Staat - Gesellschaft - Freiheit, 1976, S. 185 (199), der darin eine „Wegmarke zum Totalitarismus" erblickt.

85 Jestaedt, Demokratieprinzip (Fn. 40), S. 225 ff.

86 Grundlegend H.H. Klein, Zum Begriff der öffentlichen Aufgabe, in: DÖV 1965, S. 755 (758 f.), der sich begrifflich über Art. 30 GG nähert; weiterentwickelt und ausdifferenziert - mitunter mit verschiedenen Ansätzen, aber im Ergebnis gleich: H.P. Bull, Die Staatsaufgaben nach dem Grundgesetz, 2. Aufl. 1977, S. 11 f., 150 
Staatsgewalt im formellen Sinne ist demgegenüber die Setzung von Rechtsfolgen, mithin die autonome Entscheidung, zu verstehen ${ }^{87}$. Wichtig ist, dass die Entscheidung aus sich selbst heraus Rechtsfolgen erzeugt; „wenn eine Maßnahme zu ihrer Verbindlichkeit zwingend staatlicher Rezeption und Transformation bedarf ${ }^{\text {s }} 8$, so entfällt die Charakterisierung als Staatsgewalt. Dem pflichtet die Rechtsprechung des Bundesverfassungsgerichts bei, wenn ,jedenfalls alles amtliche Handeln mit Entscheidungscharakter ${ }^{\text {(89 }}$ als Ausübung von Staatsgewalt betrachtet wird.

\section{Das Vorzugskind Wahlfinanzierung}

Bereits die semantische Nähe von Wahlen und Abstimmungen nach Art. 20 Abs. 2 Satz 2 GG legt es nahe, die Finanzierungskonzepte dieser beiden Formen der Staatsgewaltausübung durch das Volk zu vergleichen. Darüber hinaus kann ein solcher Vergleich insbesondere in den Ländern für die Finanzierungsfrage fruchtbar gemacht werden, in denen derzeit keine Kostenerstattung vorgesehen ist. Es ist zunächst ein kurzer Überblick über das derzeitige System nach dem Parteiengesetz zu geben (1.), bevor über die Frage der Analogiefähigkeit entschieden werden kann (2.). Zum Schluss ist noch auf analogieunabhängige Konsequenzen für die Kostenerstattung für direktdemokratische Verfahren hinzuweisen (3.).

1. Das ,Ja, aber ...“ der Parteienfinanzierung

Im Gegensatz zur Kostenfrage bei direktdemokratischen Instrumenten hat die Parteien- und Wahlkampffinanzierung in gerichtlicher Praxis und juristischem Schrifttum eine intensive Zuwendung erfahren ${ }^{90}$. Die seltene Einmü-

ff.; Böckenförde, Bedeutung (Fn. 84), S. 202 f.; Jestaedt, Demokratieprinzip (Fn. 40), S. 248 ff.

87 So Jestaedt, Demokratieprinzip (Fn. 40), S. 255 ff.

88 Jestaedt, Demokratieprinzip (Fn. 40), S. 258.

89 So die Formel in BVerfGE 83, 60 (73); 93, 37 (68); in der Sache ebenso BVerfGE 47, 253 (273). Ebenso Böckenförde (Fn. 31), § 24 Rn. 12; Dreier (Fn. 19), Art. 20 (Demokratie), Rn. 90 ff.; Grzeszick (Fn. 31), Art. 20 II Rn. 91.

90 Vgl. nur die bedeutenden Entscheidungen BVerfGE 8, 51; 20, 56; 73, 40; 85, 264. Zur vielfältigen Literatur siehe die umfassenden Literaturverzeichnisse bei $U$. Volkmann Politische Parteien und öffentliche Leistungen, 1993; R. Schwartmann, Ver- 
tigkeit, mit der Parteien aller politischen Couleur ihre grundsätzliche (nicht unbedingt alle Details abdeckende) Zustimmung zu diesbezüglichen Gesetzen geben, könnten böse Zungen mit der bei der Erhöhung von Abgeordnetendiäten vergleichen. In der Rechtsprechung des Bundesverfassungsgerichts wurden in einer wechselvollen Geschichte von Verwerfung und Bestätigung auch unter mehrmaliger Aufgabe vorheriger Rechtsprechung mehrere Ansätze zur Finanzierung durchgespielt, wobei nicht selten einem Extrem das entgegengesetzte folgte ${ }^{91}$. Seinen gegenwärtigen Zustand verdankt das Parteiengesetz der Grundsatzentscheidung des Bundesverfassungsgerichts aus dem Jahre $1992^{92}$. Es nimmt nicht Wunder, dass gerade bei der Parteienfinanzierung verfassungsrechtliche Anforderungen in ihrer Ausprägung in der Rechtsprechung des Bundesverfassungsgerichts und gesetzgeberische Wirklichkeit oftmals auseinanderklaffen, entscheidet doch die parlamentarische Mehrheit regelmäßig in eigener Sache ${ }^{93}$. Da sich dieser Interessenkonflikt erfahrungsgemäß nicht über parteipolitische Selbstverpflichtungen lösen lässt, sind die verfassungsrechtlichen Maßstäbe von entscheidender Bedeutung, gerade im Hinblick auf parlamentarisch nicht vertretene Parteien.

Angesichts der grundgesetzlichen Würdigung der Parteien als Mitwirkende an der Willensbildung des Volkes in Art. 21 Abs. 1 Satz 1 GG ist prinzipiell von der staatlichen Unterstützungswürdigkeit dieser Organisationen auszugehen $^{94}$. Welche konkrete Funktion die Parteien im Rahmen des staatlichen Interaktionsprozesses der Bundesrepublik Deutschland wahrnehmen und welche Konsequenzen sich daraus für die Finanzierung ergeben, wird vom Grundgesetz nicht vorweggenommen, was Grimms Diktum von der

fassungsfragen der Allgemeinfinanzierung politischer Parteien, 1995; aus neuer Zeit siehe die Literatursammlung bei J. Ipsen, in: Sachs, GG (Fn. 43), Art. 21, S. 869 f.

91 Zur Geschichte vgl. H.H. Klein, Bundesverfassungsgericht und Parteiengesetz, in: J. Ipsen (Hrsg.), 40 Jahre Parteiengesetz, 2009, S. 19 (30 ff.); S.-C. Lenski, Parteiengesetz und Recht der Kandidatenaufstellung, 2011, § 18 PartG Rn. 14 ff.; D. Grimm, Politische Parteien, in: E. Benda/W. Maihofer/H.-J. Vogel (Hrsg.), Handbuch des Verfassungsrechts der Bundesrepublik Deutschland, 2. Aufl. 1994, § 14 Rn. 50 ff.; Ipsen (Fn. 43), Art. 21 Rn. 115 ff. Zur Reformbedürftigkeit des gegenwärtigen Systems vgl. nur M. Morlok, Parteienfinanzierung: Mängel und Reformchancen, in: H.H. v. Arnim (Hrsg.), Reform der Parteiendemokratie, 2003, S. 155 ff.

92 BVerfGE 85, 264.

93 H.H. v. Arnim, Grundfragen der Parteienfinanzierung, in: Ipsen, Parteiengesetz (Fn. 91), S. 35 (38).

94 So P. Kunig, Parteien, in: Isensee/Kirchhof, HStR III (Fn. 12), § 40 Rn. 116; $M$. Morlok, in: Dreier, GGK II (Fn. 19), Art. 21 Rn. 43. Huber, Parteien (Fn. 69), S. 614, hält indes keine Finanzierungspflicht für gegeben, obwohl die von ihm zitierte Belegstelle (BVerfGE 85, 264 [285]) eher das Gegenteil vermuten lässt. 
„,normativen Enthaltsamkeit" ${ }^{\star 95}$ begründet. Einziger Anhaltspunkt ist Art. 21 Abs. 1 S. 4 GG, der allerdings lediglich eine Offenlegungspflicht begründet und damit Privatspenden keinesfalls ausschließt, sondern vielmehr gestattet. Lag der Fokus der Funktionsbestimmung zunächst auf der Wahlvorbereitung durch die Parteien ${ }^{96}$, hat sich mittlerweile die Erkenntnis durchgesetzt, dass sich die Aufgaben der Parteien nicht darin erschöpfen ${ }^{97}$. Vielmehr besteht die Organisations- und Mittlerrolle zwischen Staat und Volk in ihrer Kontinuität unabhängig von einem spezifischen Wahltermin. Dies hat zur notwendigen Folge, dass jedenfalls eine Teilfinanzierung durch den Staat verfassungsrechtlich unbedenklich ist ${ }^{98}$. Wenn also derzeit grundsätzlich „Ja“ zu einer Parteienfinanzierung durch den Staat gesagt wird, so erfährt diese Zusage in einem nachfolgenden „Aber“ markante Einschränkungen. Aus dem Grundsatz der Staatsfreiheit des politischen Wettbewerbs leitet das Bundesverfassungsgericht $\mathrm{ab}$, dass keinesfalls der überwiegende Teil der Parteigelder aus staatlichen Mitteln bestehen darf ${ }^{99}$.

Das derzeit geltende Recht ${ }^{100}$ findet sich in $\S \S 18$ ff. PartG. Danach erhalten Parteien in Anerkennung ihrer grundgesetzlich zugeschriebenen Aufgaben (so $§ 18$ Abs. 1 S. 1 PartG) eine nach dem Wahlerfolg bei Europa-, Bundestags- und Landtagswahlen zugemessene staatliche Teilfinanzierung ( $\$ 18$ Abs. 1 S. 2 PartG). Der Betrag wird nach $\S 18$ Abs. 3 S. 1 Nr. 1 und 2 PartG auf $0,70 €$ je erhaltener Stimme pauschalisiert, wobei die ersten 4 Millionen gültigen Stimmen mit $0,85 €$ abgegolten werden ( $§ 18$ Abs. 3 S. 2 PartG). Jeder Euro, den die Parteien als private Spende erhalten, wird staatlicherseits mit $0,38 €$ bezuschusst, wobei nur Zuwendung von natürlichen Personen in

95 So Grimm, Parteien (Fn. 91), Rn. 49.

96 Etwa in BVerfGE 20, 56 (101 f.), weswegen nur eine Wahlkampfkostenerstattung für zulässig erachtet wurde.

97 BVerfGE 85, 264 (285 ff.); zu dieser Entscheidung und dem dahinterstehenden „Paradigmenwechsel“" siehe J. Ipsen, Globalzuschüsse statt Wahlkampfkostenerstattung, in: JZ 1992, S. 752 (756 f.); Schwartmann, Verfassungsfragen (Fn. 90), S. 27 ff.

98 BVerfGE 85, 264 (287 ff.); dazu und zur Funktionsbestimmung der Parteien im Allgemeinen vgl. Grimm, Parteien (Fn. 91), Rn. 12 ff.; kritisch zur Auffassung von der Mittlerrolle aber Reichel, Offenheitsprinzip (Fn. 64), S. 64 ff.

99 BVerfGE 85, 264 (287 ff.).

100 Nähere Ausführungen hierzu bei Lenski (Fn. 91), §§ 18 ff. PartG. Kritisch zur Neuregelung Kunig, Parteien (Fn. 94), Rn. 114 ff.; W. Kaltefleiter/K.-H. Naßmacher, Das Parteiengesetz 1994 - Reform der kleinen Schritte, in: ZParl 25 (1994), S. 253 (255 ff.); zu den realpolitischen Auswirkungen siehe W. Rudzio, Die Auswirkungen des neuen Parteienfinanzierungsmodells, in: ZParl 25 (1994), S. 390 (391 und pas$\operatorname{sim})$. 
Höhe von bis zu $3.300 €$ berücksichtigt werden (§ 18 Abs. 3 S. 1 Nr. 3 PartG). § 18 Abs. 4 PartG setzt spezifische Mindestgrenzen von erreichten Stimmen fest ${ }^{101}$, um überhaupt staatliche Gelder erhalten zu können. Die absolute Obergrenze an staatlichen Mitteln für alle Parteien bildet 133 Millionen Euro ( $\$ 18$ Abs. 2 PartG), die relative Obergrenze die Höhe der erzielten Spendeneinnahmen, die durch staatliche Zuschüsse nicht überholt werden darf (§ 18 Abs. 5 S. 1 PartG).

\section{Wie ähnlich sind sich Wahl und Abstimmung?}

Nach dieser kurzen Darstellung des geltenden Rechts der Parteienfinanzierung bleibt die Frage, welche dieser Regelungen auf direktdemokratische Verfahren auszuweiten sind. Die rechtswissenschaftlich herausgearbeiteten Voraussetzungen der Analogie ${ }^{102}$, eine planwidrige Regelungslücke bei gleichzeitiger Interessenähnlichkeit, sind auf das Verhältnis von Wahl und Abstimmung anzuwenden.

\section{a) Das Schweigen der Gesetze}

Eine planwidrige Regelungslücke ist nur dann anzunehmen, wenn der Gesetzgeber nicht bewusst von einer Regelung abgesehen hat. Auch eine bewusste Nichtregelung, die gerade eine Kostenerstattung in direktdemokratischen Verfahren ausschließen soll, stellt eine zu beachtende Entscheidung des Gesetzgebers dar. Hat also ein Landesgesetzgeber sich zu einer gesetzlichen Fixierung - sei sie auch teilweise - entschlossen, verbleibt kein Raum für eine analoge Anwendung der Wahlkampfkostenerstattung. Eine Regelungslücke kann somit von vornherein nur in denjenigen Ländern bestehen, die sich zu dieser Frage gesetzlich überhaupt nicht geäußert haben. Wegen der sachlichen Nähe des Wahl- und des Abstimmungskampfes und des gleichgearteten Finanzierungsbedarfes liegt es prima facie nahe, diese beiden

101 Z.B. eine Erfolgsquote von 0,5\% für die Europa- und Bundestagswahl bzw. 1\% für die Landtagswahlen sowie von 10\% für die in einem Wahl- oder Stimmkreis abgegebenen Stimmen.

102 Siehe nur die klassische Darstellung bei K. Larenz, Methodenlehre der Rechtswissenschaft, 6. Aufl. 1991, S. 381 ff.; sowie die Ausführungen bei F. Bydlinski, Juristische Methodenlehre und Rechtsbegriff, 2. Aufl. 1991, S. 475 ff.; K.F. Röhl/H.C. Röhl, Allgemeine Rechtslehre, 3. Aufl. 2008, S. 633 ff. 
Materien parallel $\mathrm{zu}$ regeln. Allerdings finden sich in den entsprechenden Gesetzen Bestimmungen über die Verfahrenskosten, die die Kostenverteilung zwischen Land und Gemeinden zum Gegenstand haben. Daraus könnte der Schluss zu ziehen sein, dass die Länder wissentlich auf eine Kostenerstattung für Abstimmung verzichtet haben ${ }^{103}$. Jedoch spricht bereits eine numerische Betrachtung gegen diese Auffassung. Die Verfahren der unmittelbaren Demokratie werden im Vergleich zu den regelmäßig wiederkehrenden Wahlverfahren selten angewandt und wenn, dann steht nicht die Refinanzierung des Abstimmungskampfes im Mittelpunkt der gesellschaftlichen und gesetzgeberischen Debatte ${ }^{104}$. Weiterhin stehen hinter privaten Initiativen manchmal parlamentarische Oppositionsparteien, sodass Finanzierungsfragen über diese wirtschaftlich potenten Organisationen abgewickelt werden $^{105}$. Dadurch bleibt die Abstimmungskampfkostenerstattung außerhalb der parlamentarischen Diskussion. Zuletzt regeln die entsprechenden Landesgesetze die Wahlkampfkostenerstattung und die Kostentragung des Wahlverfahrens systematisch voneinander getrennt, weshalb die parallelen Verfahrensregelungen zu Abstimmungen nicht weiter ins Gewicht fallen. Demnach lässt sich aus der bloßen gesetzlichen Nichtregelung keineswegs ein bewusstes Unterlassen ableiten.

\section{b) Der Gleichlauf der Interessen}

Ein schlichtes Vergessen seitens des Gesetzgebers berechtigt aber für sich genommen noch nicht den Rechtsanwender, ein anderes Rechtsinstitut an die Stelle der Gesetzeslücke zu setzen. Hinzukommen muss außerdem eine hinreichende Ähnlichkeit, insbesondere hinsichtlich der involvierten Interessen. Die Rechtsprechung bietet ein vielfältiges Meinungsbild, das je nach sachlichem Zusammenhang von partikularen Übereinstimmungen ${ }^{106}$ oder fundamentalen Gegensätzen ${ }^{107}$ ausgeht. Aufwendige und wegen ihrer verfas-

103 So zum alten Landesrecht, das aber insoweit mit dem neuen übereinstimmt: StGH BW ESVGH 22, 1 (2 f.).

104 So auch Przygode, Rechtsprechung (Fn. 6), S. 458 f., der von einer „Spekulation“ des StGH BW spricht, die ,,jeglicher Grundlage und Überzeugungskraft" entbehre.

105 Przygode, Rechtsprechung (Fn. 6), S. 458.

106 Etwa VGH München, NVwZ 1991, S. 699 (700 f.).

107 BayVerfGH, BayVB1. 1994, S. 203 (205 f.); beipflichtend W. Schmitt-Glaeser/H.-D. Horn, Die Rechtsprechung des Bayerischen Verfassungsgerichtshofs, in: BayVB1. 1994, S. 289 (296 ff.); ebenso, aber ohne nähere Begründung BVerwG, NVwZ 1983, S. 737 (737). 
sungsmäßigen Stellung bedeutsame Verfahren wie Wahlen und Abstimmungen bedürfen einer ausdifferenzierten gesetzlichen Regelung, die sich auf die verschiedenen Bereiche von behördlicher Neutralität bis zu Anfechtungsfristen erstreckt. Eine Vergleichbarkeit von Interessen kann somit nur in Bezug auf ein spezifisches Regelungsinstrument festgestellt werden. Die in anderem Zusammenhang vorgetragenen Argumente sind demnach nicht unreflektiert übertragbar. Vielmehr müssen sie aus dem Blickwinkel der konkreten Anforderungen und Besonderheiten der Kostenerstattung betrachtet werden.

Hauptsächliches Argument des Bayerischen Verfassungsgerichtshofes in seinem Urteil aus dem Jahr $1994^{108}$ ist der angenommene Funktionsunterschied von Wahl und Volksentscheid. In dieser Entscheidung ging es um eine Veröffentlichung der Bayerischen Staatsregierung zu einem Volksentscheid. Die Wahl diene in erster Linie der demokratischen Legitimation der Ausübung von Staatsgewalt ${ }^{109}$. Demgegenüber sei im Volksentscheid über eine Sachfrage in der Rolle des Gesetzgebers zu entscheiden ${ }^{110}$. Staatliche Öffentlichkeit seitens der Landesregierung sei aber im Gesetzgebungsprozess - anders als bei der Wahl ${ }^{111}$ - nicht grundsätzlich untersagt. Daher könne dieser Wahlrechtsgrundsatz nicht ohne Weiteres auf eine Abstimmung übertragen werden ${ }^{112}$. Unabhängig von der Tatsache, dass mit dieser Funktionszergliederung die einheitliche Rückanbindung von Wahlen und Abstimmungen als Ausübung von Staatsgewalt in Art. 20 Abs. 2 S. 2 GG negiert wird, kann dieses Argument im Rahmen der Kostenerstattung nicht durchgreifen. Schließlich ist mit einer abweichenden Aufgabenbestimmung keine Aussage über die staatliche Unterstützungswürdigkeit getroffen. Mandatsträger etwa werden auch und gerade wegen ihrer Beteiligung am Gesetzgebungsprozess staatlich alimentiert. Gerade in der Anerkennung des Volksentscheides als Gesetzgebungsverfahren liegt eine Einordnung als unmittelbares staatliches Geschehen, das dem rein Privaten enthoben ist und wegen ebendieser Nähe zu genuin staatlichen Aufgaben für öffentliche Gelder zumindest geeignet erscheint.

Die $\S \S 18$ ff. des Parteiengesetzes sind jedoch aus ihrem systematischen Kontext heraus zu verstehen. Als Reaktion des Gesetzgebers auf die Ent-

108 BayVerfGH, BayVBl. 1994, S. 203 ff.; detaillierter zu dieser Entscheidung H. Holzheid, Maßgebliche Verfassungsgrundsätze bei Wahlen und Volksbegehren, 1995, S. $26 \mathrm{ff}$.

109 BayVerfGH, BayVB1. 1994, S. 203 (205).

110 BayVerfGH, BayVB1. 1994, S. 203 (205).

111 Vgl. BVerfGE 44, 125 (151 ff.); 63, 230 (24).

112 BayVerfGH, BayVB1 1994, 203 (205). 
scheidung des Bundesverfassungsgerichts von 1992 kann das Regelungskonzept nur korrekt eingeordnet werden, wenn die dort getroffenen verfassungsrechtlichen Weichenstellungen mit einbezogen werden. Lässt sich „die Tätigkeit der Parteien im Wahlkampf von ihrer sonstigen Tätigkeit“ nur „,rein äußerlich“ abgrenzen und beschränkt sich ,die den Parteien in Art. 21 Abs. 1 Satz 1 GG aufgegebene Mitwirkung bei der politischen Willensbildung des Volkes [...] nicht auf die unmittelbare Wahlvorbereitung “"113, dann geht das gegenwärtige System der Parteienfinanzierung über die bloße Wahlkampfkostenerstattung hinaus. Die durch Art. 21 Abs. 1 S. 1 GG herausgehobene verfassungsrechtliche Position der Parteien ist nur unzureichend mit einer Initiatorengruppe zu vergleichen; der entscheidende Unterschied lässt sich mit der Gegenüberstellung von Kontinuität und Singularität markieren. Vielmehr zeigt bereits die Regelung der Kostenmaterie im Parteienrecht (und nicht etwa im Bundeswahlgesetz), dass sie nicht unmittelbar dem regulären Wahlrecht zugeordnet werden kann. Sie beruht in ihrer gegenwärtigen Ausgestaltung auf der spezifisch parteienrechtlichen Disposition der Wahlkampfkostenerstattung ${ }^{114}$. Demnach sind die Normen zur Parteienfinanzierung nicht im Wege einer Analogie auf die Kostenerstattung von direktdemokratischen Abstimmungskämpfen zu übertragen.

\section{Bedeutung abseits der Analogie}

Auch wenn eine Analogie an durchgreifenden Bedenken scheitert, bleibt die Art und Weise der Regelung der Parteienfinanzierung nicht ohne Folgen für die Abstimmungskostenerstattung. Das System der Parteienfinanzierung erkennt in seiner gegenwärtigen Ausprägung die Parteien in ihrer Mittlerrolle zwischen Staat und Gesellschaft an ${ }^{115}$ und weist ihnen aus diesem Grund staatliche Mittel zu. Die Parteien verfolgen demnach keinen von der demokratischen Konstitution des Gemeinwesens abgekoppelten Selbstzweck, sondern wirken bei der Verwirklichung des Prinzips der Volkssouveränität nach Art. 20 Abs. 2 S. 1 GG mit. Staatliche Finanzierung hat sich in Anlehnung an diese verfassungsrechtliche Zuweisung letztlich an der Sicherung und Reali-

\section{BVerfGE 85, 264 (286).}

114 So auch Przygode, Rechtsprechung (Fn. 6), S. 459.

115 BVerfGE 52, 63 (82 f.); wiederholt in E 60, 53 (66 f.); K. Hesse, Die verfassungsrechtliche Stellung der politischen Parteien, in: VVDStRL 17 (1959), S. 11 (19), spricht von der „Funktion eines Bindegliedes zwischen Volk und politischer Führung". 
sierung ebendieser Souveränität zu orientieren ${ }^{116}$. Lässt sich die Willensbildung des Volkes durch die Modalitäten der staatlichen Finanzierung nicht mehr in der gebotenen Staatsfreiheit vollführen, greifen die verfassungsrechtlichen Gewährleistungen des Demokratieprinzips. Die dienende und - in der historischen Entwicklung immer bedeutungsvollere - gestaltende Funktion der Parteien ist seitens des Staates durch eine angemessene Unterstützung anzuerkennen. Begründungsmuster und Legitimitätsquelle ist aber stets die Souveränität des Volkes, deren realgesellschaftliche Durchsetzung in einer repräsentativen Demokratie auf Parteiarbeit angewiesen ist ${ }^{117}$. In der Rückführung auf diesen Ursprungsquell lassen sich daher auch Maßstäbe für die direktdemokratischen Instrumente gewinnen. Denn in der Abstimmung partizipiert der Bürger in unmittelbarer Weise am Staatswillen, ohne auf die Mittlerrolle der Parteien angewiesen zu sein. Gleichzeitig werden in der Abstimmung zwar die Parteien als Scharnier zwischen Staat und Gesellschaft in institutioneller Hinsicht obsolet, jedoch gilt dies nicht in demselben Maße für deren Funktion als Vermittler. Auch vor einer Abstimmung bleibt das Bedürfnis, die Öffentlichkeit über Vor- und Nachteile des Vorschlags zu informieren. Die Wettkampfsituation, in der sich Parteien in Zeiten des Wahlkampfes und vermindert auch dazwischen befinden ${ }^{118}$, kehrt in Grundzügen bei der Abstimmung wieder. Zwar geht es, anders als bei Wahlen, nicht um Personal- und Ämterentscheidungen, doch ist eine Sachfrage keineswegs weniger kontrovers und diskurswürdig. Im denkwürdigen Streit um Auslegung und Anwendung des Grundgesetzes im Bereich der Parteienfinanzierung lässt sich jedenfalls festhalten, dass die verfassungsrechtliche Zuerkennung auf der Grundsatzentscheidung des Art. 20 Abs. 1 und 2 GG beruht. Die Analyse des Systems der Parteienfinanzierung zeigt, dass Volkssouveränität und nicht schlichter Kostendeckungsbedarf der in Art. 21 Abs. 1 GG anerkannten Parteien den dogmatischen Sockel bildet, auf dem das Rechtsinstitut ruht. Volkssouveränität findet ihren Ausdruck aber auch und gerade in den direktdemokratischen Verfahren.

116 N. Achterberg/M. Schulte, in: v. Mangoldt/Klein/Starck, GG II (Fn. 48), Art. 38, Rn. 150, begründen so den verwandten Grundsatz der Chancengleichheit, an dem sich staatliche Finanzierung ebenfalls messen lassen muss.

117 Zum Zusammenhang von Demokratieprinzip und Parteienfreiheit vgl. auch Hesse, Stellung (Fn. 115), 17 ff.; Morlok (Fn. 19), Art. 21 Rn. 19 ff.

118 Vergleiche zu diesem Topos nur die beiden Beiträge auf der Staatsrechtslehrertagung 2009: A. Hatje, Demokratie als Wettbewerbsordnung, in: Veröffentlichungen der Vereinigung der Deutschen Staatsrechtslehrer 69 (2009), S. 135 ff., insbs. S. 151 ff.; M. Kotzur, ebda., S. 173 ff., hier besonders S. 191 ff. sowie S. 206 ff. 
V. Direkte Demokratie als gehobenes Hobby oder das faktische Kostenbedürfnis

Bevor die oben (III.) entwickelten rechtlichen Parameter auf die Gesetzeslage angewendet werden, bietet es sich an, einen Blick auf den tatsächlichen Finanzaufwand für direktdemokratische Instrumente zu werfen sowie die Auswirkungen des Geldeinsatzes auf den Verfahrensausgang zu beleuchten.

1. Kostenzwänge als rechtliches Kriterium

Zunächst sind die zu erwartenden Kosten eines Verfahrens der unmittelbaren Demokratie gerade für die Initiatoren unter dem Gesichtspunkt der Prognose und Kalkulation bedeutsam. Dieses privat-pekuniäre Interesse erstarkt aber zu einem Problem von objektiv-rechtlicher Relevanz, wo der Kostenfaktor maßgebenden Einfluss auf den Ablauf und Ausgang eines direktdemokratischen Prozesses ausübt. Dann nämlich tritt die Schutzfunktion des Gesetzgebers (siehe unter III. 2.) in den Vordergrund und verpflichtet zu regulativen Eingriffen. Wo die Grenze zwischen hinzunehmenden Ungleichgewichten und wettbewerbsverzerrenden Asymmetrien verläuft, lässt sich durch bloß deskriptives Anführen des Kostenbedarfs nicht bestimmen. Auch ist bei einer solch normativen Frage der empirisch-messbare Finanzierungsaufwand nicht ausschließliches Kriterium. Aber er kann zumindest indizielle Wirkung entfalten, die sich dann in der rechtlichen Bewertung widerspiegelt. Die Wertung selbst ist allerdings - im Rahmen verfassungsrechtlicher Grenzen - dem Gesetzgeber vorbehalten.

\section{Kosten der direktdemokratischen Instrumente}

a) Einfluss des Geldes als wissenschaftliches weißes Blatt

Bislang war der tatsächliche Finanzierungsaufwand von direktdemokratischen Institutionen kaum Gegenstand von wissenschaftlichen Untersuchungen. Über die Gründe dieser empirischen terra incognita kann nur spekuliert werden. Naheliegend erscheint dabei der Umstand, dass direktdemokratische Verfahren nicht allzu zahlreich gesät sind bzw. dass ein unterfinanziertes Verfahren bereits von vornherein keine - gesellschaftliche oder wissen- 
schaftliche - Aufmerksamkeit erfährt. Weiterhin ist zu beachten, dass sich die Initiatoren entweder als institutionell unterstützte Interessengruppen ${ }^{119}$ oder als partikular mobilisierte „Aktivbürger“ darstellen. Erstgenannte bleiben ohnehin gern im Hintergrund und sind in der Regel finanziell gut ausgestattet, Letztere kompensieren ihre vergleichsweise wirtschaftliche Schwäche durch Enthusiasmus und Breitenwirkung. In Wahlkämpfen spielen Reformen von direktdemokratischen Instrumenten nur eine untergeordnete Rolle, und während einer Legislaturperiode ist dieses Thema erfahrungsgemäß nicht von vordringlicher politischer Bedeutung.

\section{b) Erwägungen zum Finanzierungsbedarf in Deutschland}

Der Finanzierungsbedarf direktdemokratischer Instrumente unterscheidet sich naturgemäß je nach Verfahrensart. Beschränken sich die Initiatoren nur auf die gesetzlich vorgeschriebenen Mindestanforderungen, so stellen sich die Volksinitiative und das Volksbegehren als am kostspieligsten heraus. Schließlich muss hier ein gewisses Quorum an Unterstützerunterschriften erreicht werden. Sollte das Ergebnis hinter der Mindestanzahl zurückbleiben, ist die Initiative oder das Begehren gescheitert. Je größer und bevölkerungsreicher das Bundesland, desto aufwendiger ist die Beschaffung der Unterschriften. In Nordrhein-Westfalen, Bayern oder Baden-Württemberg etwa ist es kaum möglich, das erforderliche Minimum an Unterschriften nur durch persönlichen Einsatz zu gewinnen. Kann sich die Initiatorengruppe also nicht auf einen außerordentlich großen Unterstützerkreis verlassen, müssen gewerbliche Unterschriftensammler eingesetzt werden.

Für den Volksentscheid werden hingegen nur Bürgerstimmen benötigt, die an sich keine Kosten verursachen. Es ist jedoch unmittelbar einsichtig, dass für einen Volksentscheid eine ausreichende Publizität hergestellt werden muss, ohne die die Mindestanzahl an Ja-Stimmen nicht erreicht werden kann. Nur in den allerwenigsten Fällen wird diese öffentliche Aufmerksamkeit allein durch die Berichterstattung unabhängiger Medien generiert. Hinzukommt, dass regelmäßig die widerstreitenden Positionen auch entgegengesetzte wirtschaftliche Ziele verfolgen. Begnügt sich die Initiatorengruppe mit der Werbung über kostenfreie, aber weniger effektive Kanäle, kann die opponierende Seite durch gezielten Mitteleinsatz die öffentliche Meinung beeinflussen. Potenziert wird dieser Effekt durch eine wirkmächtige Finanzaus-

119 Siehe näher bei Przygode, Rechtsprechung (Fn. 6), S. 451. 
stattung, wie sie üblicherweise wirtschaftsnahen Kreisen - jedenfalls bei bedeutenden Abstimmungen - zur Verfügung steht.

Konkrete Zahlen ${ }^{120}$ lassen sich so naturgemäß nicht gewinnen, hier sind weitere wissenschaftliche Studien gefragt. Dennoch sind Kostenzwänge im Bereich der direkten Demokratie auch ohne überbordende Phantasie nachvollziehbar, angesichts der Erkenntnisse aus dem Wahlkampf lässt sich zum jetzigen Zeitpunkt feststellen, dass der ,für die Praxis äußerst bedeutsame Aspekt der Kosten“"121 keinesfalls unterschätzt werden darf.

\section{Politikwissenschaftliche Studien zur Entscheidungserheblichkeit}

Wissenschaftliche Untersuchungen zum Einfluss von Geldmitteln und Werbung in Abstimmungskämpfen sind hauptsächlich in den USA und der Schweiz angestellt worden ${ }^{122}$. Die empirisch besser gesicherten Ergebnisse lassen sich allerdings in den USA ermitteln, weil die dort teilweise geltenden Offenlegungspflichten von Spenden die konkret zur Verfügung stehenden Geldbeträge öffentlich machen ${ }^{123}$. Im Umfang dieser Arbeit kann keine Dis-

120 Eine kurze Darstellung von Abstimmungskosten ohne Anspruch auf Vollständigkeit oder Prognosefähigkeit unternimmt Jung, Grundsatzfragen (Fn. 11), S. 332 f. Die dargestellten Verfahren schwanken in den Kosten zwischen 200.000 und 600.000 DM. Von den Kosten für zwei Volksbegehren aus den Jahren 1973 bzw. 1976 in Höhe von 1 bzw. 1,2 Millionen DM berichtet ders., Regeln (Fn. 7), S. 54. In neuerer Zeit finden sich Übersichten bei dems., Direkte Demokratie in Berlin. Der Fall „Pro Reli“ 2007-2009, Berlin 2011, S. 120 ff.; dort führt Jung den Finanzierungsaufwand von fünf direktdemokratischen Verfahren auf, konstatiert aber gleichfalls insgesamt „wenig empirisches Material“. Ein weiteres Beispiel (Nichtraucherschutz in Bayern) führt B.M. Weixner, Nichtraucherschutz in Bayern - der Weg eines erfolgreichen Volksbegehrens und Volksentscheids im Freistaat, L.P. Feld/P.M. Huber/O. Jung/C. Welzel/F. Wittreck (Hrsg.), Jahrbuch für direkte Demokratie 2010, 2011, S. 255 (284 f.). Die angegebenen Summen bewegen sich jeweils im Bereich von mehreren hunderttausend Euro. Eine Übersicht über die Schweizer Verhältnisse findet sich bei auch M. Caroni, Geld und Politik. Die Finanzierung politischer Kampagnen im Spannungsfeld von Verfassung, Demokratie und politischem Willen, 2009, S. 27 ff., dort werden nationale Referenden mit bis zu 7,5 Millionen Franken veranschlagt. D.A. Smith, US States, in: K. Gilland Lutz/S. Hug (Hrsg.), Financing Referendum Campaigns, 2010, S. 39 (45), spricht gar von 398 Millionen US-\$, die 2004 in allen US-Staaten für direktdemokratische Verfahren ausgegeben wurden.

121 Przygode, Rechtsprechung (Fn. 6), S. 451.

122 Eine kurze Darstellung anhand eines konkreten Falles aus Deutschland findet sich bei Jung, Berlin (Fn. 120), S. 152 ff.

123 In diese Richtung auch Caroni, Geld (Fn. 120), S. 42. 
kussion zur Methodik dieser Studien erfolgen ${ }^{124}$. Auch wenn im Einzelnen viele Schlussfolgerungen umstritten oder unklar sind, lässt sich zumindest eine merkliche Korrelation von Geldeinsatz und Abstimmungserfolg konstatieren $^{125}$. Die aktuelle und umfassende Studie von $J$. de Figueiredo, C. H. Ji und T. Kousse analysiert die Referenden der Jahre 1978 bis 2004 in Kalifornien und kommt zu dem Ergebnis, „both support and opposition spending on initiatives exert strong and statistically significant effects on their chances of passage. “ ${ }^{126}$ H. P. Hertig formuliert den Einfluss auf den Abstimmungserfolg noch schärfer: „Eine gute Kaufchance ist [...] unseres Erachtens im allgemeinen gegeben. “127

124 Zur schwierigen Unterscheidung von Kausalität und Korrelation und weiteren empirischen Ungewissheiten siehe T. Stratmann, The Effectiveness of Money in Ballot Measure Campaigns, in: Southern California Law Review 78 (2005), S. 101 (101 ff., $110 \mathrm{ff}$.).

125 Einen leichten Effekt stellen fest T. Stratmann, Campaign Spending and Ballot Measures, in: Gilland Lutz/Hug, Campaigns (Fn. 120), S. 9 (19 f.), der darauf verweist, dass frühere Studien (darunter muss er seine eigene [Fn. 124] fassen) prägnantere Auswirkungen angenommen hatten; U. Serdült, Referendum Campaign Spending Regulations in Switzerland, ebda., S. 165 (171 ff.). Stärkere Auswirkungen beobachten hingegen R. Mastro/D.C. Costlow/H.P. Sanchez, Taking the Initiative: Corporate Control of the Referendum Process Through Media Spending and What to Do About It, in: Federal Communications Law Journal 32 (1980), S. 315 (319 ff.); Stratmann, Effectiveness (Fn. 124), S. 123 f. Die unterschiedlichen Ergebnisse sieht Smith, States (Fn. 120), S. 52 ff., in den andersartigen Bedingungen je nach Bundesstaat begründet. Einen guten Überblick über die US-Verhältnisse gibt H.K. Heußner, Direkte Demokratie in den US-Gliedstaaten im Jahr 2008, in: L.P. Feld/P.M. Huber/O. Jung/C. Welzel/F. Wittreck (Hrsg.), Jahrbuch für direkte Demokratie 2009, 2010, S. 165 (196 ff.) sowie ders., Direkte Demokratie in den US-Gliedstaaten in den Jahren 2010 und 2011 - Ein Überblick, in: L.P. Feld/P.M. Huber/O. Jung/C. Welzel/F. Wittreck (Hrsg.), Jahrbuch für direkte Demokratie 2012, 2013, S. 181, (217 f.); Heußner bemängelt eine ,zu starke Dominanz“ des Geldes.

126 J. de Figueiredo/C.H. Ji/T. Kousser, Financing Direct Democracy: Revisiting the Research on Campaign Spending and Citizen Initiatives, in: The Journal of Law, Economics, \& Organization 27 (2011), S. 485 (508).

127 H.P. Hertig, Sind Abstimmungserfolge käuflich?: Elemente der Meinungsbildung bei eidgenössischen Abstimmungen, in: Schweizerisches Jahrbuch für Politische Wissenschaft 22 (1982), S. 35 (56). Ausgewogener formuliert allerdings in neuerer Zeit H. Kriesi, Sind Abstimmungsergebnisse käuflich?, in: A. Vatter/F. Varone/F. Sager (Hrsg.), Demokratie als Leidenschaft. Festschrift für Wolf Linder, 2009, S. 83 (104), der einen zwar relativ geringen, aber unter Umständen entscheidenden Einfluss ausmacht. 


\section{Kurzschluss der Übertragbarkeit}

Wissenschaftliche Studien belegen also in konstanter und beachtlicher Weise den Einfluss von finanziellen Mitteln auf den Ausgang direktdemokratische Verfahren. Es ist hierbei jedoch zu beachten, in welchem politischen und gesellschaftlichen Klima diese Untersuchungen ihr Datenmaterial gewonnen haben. Insbesondere in Kalifornien und der Schweiz im Generellen sind Verfahren der unmittelbaren Demokratie sowohl institutionell als auch personell stärker verankert als in Deutschland. Dort sind also regelmäßig quasiprofessionelle Interessengruppen und Bürgerzusammenschlüsse aktiv, die unter anderen Vorbedingungen operieren als die meisten deutschen InitiativGruppierungen. Die unter diesen Voraussetzungen ermittelten Ergebnisse sind daher nicht per se als allgemeingültige Abstimmungssoziologie zu begreifen. Dennoch lassen die kontinuierlich und konsentiert erzielten Schlussfolgerungen einen parallelen Ausgang in einer deutschen Studie plausibel erscheinen. Die in amerikanischen und schweizerischen Untersuchungen festgestellten und belegten finanziellen Beeinflussungen des Abstimmungsergebnisses sind daher nicht zwingend, aber mit großer Wahrscheinlichkeit auch in Deutschland vorhanden.

VI. Verfassungsanspruch und Verfassungswirklichkeit im Hinblick auf die Kostenfrage

Es bleibt nun, die gegenwärtige Rechtslage in den Ländern an den so entwickelten Folgerungen aus dem Demokratieprinzip zu messen. Dabei bietet sich eine Aufteilung nach den oben ${ }^{128}$ dargestellten Kategorien der Finanzierung an. Baden-Württemberg und Rheinland-Pfalz werden dabei so behandelt, als ob es in diesen Ländern überhaupt keine Kostenerstattung gäbe ${ }^{129}$.

\section{Kostenerstattung für den Volksentscheid}

Der Volksentscheid, also die Endstufe der Volksgesetzgebung, zeichnet sich durch unmittelbare Einwirkung auf die Rechtsordnung eines Landes aus. Durch den Volksentscheid wird verbindlich darüber entschieden, ob ein

128 Unter II.7.

129 Vgl. oben II.9.c. 
Entwurf Gesetz sein soll oder nicht. Diese Entscheidung ist daher unmittelbar der staatlichen Sphäre zuzuordnen und dem eigentlich privaten Bereich enthoben ${ }^{130}$. Veranschaulicht wird dies dadurch, dass wie bei der Wahl das gesamte Stimmvolk zur Stimmabgabe aufgerufen ist und die Initiatoren am Abstimmungsprocedere im engeren Sinne nicht teilnehmen. Vielmehr wird in analoger Weise zur Wahl die Durchführung von staatlichen Stellen übernommen, und nur der Abstimmungskampf liegt in selbständiger Verantwortung privater Personen. Gesetzlich ist somit keinerlei private Mitwirkung geboten - sie ist lediglich im Sinne des Abstimmungserfolges zweckmäßig, was den Vorgang weiterhin als staatliches Geschehen auszeichnet.

Schon nach dem eindeutigen Wortlaut des Art. 20 Abs. 2 S. 2 GG liegt hier also ein Fall staatlicher Willensbildung vor ${ }^{131}$, für den die Anforderungen des Demokratieprinzips uneingeschränkt gelten. Auch liegt hier eine größere sachliche Nähe zur Wahlkampfkostenerstattung vor, als dies bei Volksinitiative und Volksbegehren der Fall ist. Damit muss, auch unabhängig von der verfassungsrechtlichen Wertentscheidung des Art. 21 Abs. 1 GG, im Mindesten die Finanzierungsfähigkeit angenommen werden. Wegen der Einflüsse von Abstimmungskampagnen, die sich ihrerseits durch einen großen Geldbedarf auszeichnen ${ }^{132}$, und der unmittelbaren Zuordnung zu staatlichen Sphäre ist aber darüber hinaus eine teilweise Finanzierungspflicht des Staates festzustellen. Das empirische Material, das durch die Entscheidung des (Bundes-)Gesetzgebers zur Parteienfinanzierung zumindest beim Wahlkampf rezipiert wurde, legt potentiell verzerrende Einflüsse nahe.

Eine solche Kostenerstattung begegnet auch nicht dem bei der Parteienfinanzierung erhobenen Einwand, dadurch werde die einmal entstandene parteipolitische Landschaft auf Dauer zementiert, indem nämlich der zurückliegende Erfolg belohnt wird. Initiatoren eines direktdemokratischen Verfahrens sind häufig partikulare Interessengruppen, die nicht in einem kontinuierlichen Prozess an der staatlichen Willensbildung teilhaben. Für die opponierende Gruppierung, die durchaus aus ,arrivierten“ politischen Akteuren wie etwa Parteien bestehen kann, gebietet es aber zumindest beim Volksentscheid der Grundsatz der Chancengleichheit ${ }^{133}$, auch hier Kosten zu erstatten. Jedenfalls beim derzeitigen Zustand sowohl der Parteien als auch der direkt-

130 Deutlich Jung, Grundsatzfragen (Fn. 11), S. 332: „Volksgesetzgebung ist kein Privatvergnügen" (Hervorhebung im Original).

131 Ebenso Hartmann, Volksgesetzgebung (Fn. 83), S. 102 ff., der dies in Anlehnung an das Gesetzgebungsverfahren der Art. 76 ff. GG noch weiter ausführt.

132 Dazu oben V.2.

133 Hierzu oben III.2. 
demokratischen Verfahren ist es nicht wahrscheinlich, dass Parteien durch massive Geldmittel, die dann nicht mehr für den Wahlkampf zur Verfügung stehen, den Abstimmungskampf verzerren. Eine Gefährdung des chancengleichen demokratischen Prozesses geht hier eher von wirtschaftsnahen Kreisen aus. Sobald beide Seiten auf einen Topf von staatlichen Geldern zugreifen können, herrscht zumindest in der Ausgangssituation nahezu Waffengleichheit. Darum ist auch eine „Pseudokostenerstattung“ wie in Sachsen $(0,0102 €$ pro Stimme) oder Thüringen $(0,0075 €$ pro Stimme) fragwürdig. Ein solcher Betrag kann nicht einmal ansatzweise die Kosten des Abstimmungskampfes decken und kommt funktionell einer gänzlich fehlenden Kostenerstattung gleich. Bayern, Berlin, Brandenburg, Bremen, Hessen, Mecklenburg-Vorpommern, Nordrhein-Westfalen und das Saarland stehen demgegenüber in der Pflicht, überhaupt die Kostenerstattung für den Volksentscheid vorzusehen. Für Baden-Württemberg und Rheinland-Pfalz gilt dies wie bereits festgestellt ${ }^{134}-$ im Wesentlichen auch.

\section{Kostenerstattung für die Volksinitiative}

Zunächst ist die Frage zu behandeln, ob bereits für die Initiativphase eine Kostenerstattung stattfinden muss. Dies ist wesentlich von der Funktion dieser Verfahrensart im staatlichen Gefüge der Länder aus zu beurteilen. Die Volksinitiative zielt zunächst nur darauf ab, das Parlament mit einer für bedeutsam gehaltenen politischen Frage zu befassen ${ }^{135}$. Unmittelbare Rechtswirkung entfaltet daher auch die zustande gekommene Initiative nicht. Dies gilt auch für die Länder, die ein zweistufiges Verfahren vorsehen, dort kommt dem Antrag auf Zulassung eines Volksbegehrens ebenfalls keine Entscheidungsqualität $\mathrm{zu}^{136}$. Geht es in der Sache also noch um das Parlament als Gesetzgebungsorgan, das in nicht verpflichtender Weise auf einen potentiellen Gesetzgebungsgegenstand (oder eine sonstige politische Frage) hingewiesen wird, so ist dies im Wesentlichen der gesellschaftlichen Sphäre zuzuordnen. Es handelt sich hierbei um den zwar rechtlich ausgestalteten, aber im Kern doch unverbindlichen Versuch, das Parlament mit einem Problem zu befassen.

134 Zur dort bereits bestehenden „Kostenerstattung“" siehe oben II.9.c.

135 Z.B. in Hamburg: Art. 50 Abs. 1, 2 HmbLV; Niedersachsen: Art. 47 NdsLV; Nordrhein-Westfalen: Art. 67a Abs. 1 S. 1 LV NW; Sachsen-Anhalt: $§ 9$ Abs. 1-3 VAbstG LSA.

136 Als Beispiel sei hier nur Thüringen genannt, Art. 82 Abs. 3, 5 ThürLV. 
Etwas anderes könnte sich allenfalls aus der Tatsache ergeben, dass auch in der Initiativphase beträchtliche Kosten durch das Unterschriftensammeln auf die Initiatoren zukommen. Aber weder das empirische Material, das sich hauptsächlich auf den Abstimmungskampf und die Werbungskosten konzentriert, noch eine normative Betrachtung der Staatsnähe der Aktivität zeigen für diese Form der unmittelbaren demokratischen Verfahren unabweisbare Sachzwänge oder Wertungsgesichtspunkte auf, die eine staatliche Finanzierung unumgänglich machen.

Zuletzt sollte auch die Filterfunktion der Volksinitiative nicht vernachlässigt werden. Sie trennt bürgerschaftliche Anliegen, die umfassendere Diskussionswürdigkeit und Bedeutung in sich tragen, von solchen, die nicht in erforderlichem Maße relevant erscheinen. Natürlich ist die Finanzausstattung eines Bürgerbegehrens allein noch kein hinreichendes Kriterium für seine politische Notwendigkeit, allerdings sollte bei dieser Verfahrensstufe die potentielle Vermögenslosigkeit der Initiatoren noch durch individuelles Engagement aufgewogen werden können. Sofern dieses jedoch nicht vorhanden ist, darf man durchaus an der Dringlichkeit des Anliegens zweifeln.

\section{Kostenerstattung für das Volksbegehren}

Von anderer rechtlicher Qualität sind die Volksbegehren. Hier besteht zwar auch zunächst nur eine Befassungspflicht des Parlaments ${ }^{137}$, doch kann im Falle der Ablehnung ein Volksentscheid verlangt werden ${ }^{138}$. Funktionell stellt das Volksbegehren eine eigenständige Form des Gesetzgebungsinitiativrechts dar ${ }^{139}$. Allerdings trifft das Volksbegehren noch keine abschließende Entscheidung, sondern dringt im Gegenteil auf eine Entscheidung des

137 Das Volksbegehren ist generell so ausgestaltet, vgl. nur Baden-Württemberg: Art. 59 Abs. 2 S. 3 LV BW; Bayern: Art 74 Abs. 3 BayLV; Hamburg: Art 50 Abs. 3 S. $1 \mathrm{HmbLV}$; Nordrhein-Westfalen: Art. 68 Abs. 2 S. 1 LV NW; Sachsen-Anhalt: Art. 81 Abs. 2 S. 2 LV LSA; Thüringen: Art. 82 Abs. 7 S. 1 ThürLV.

138 Ebenfalls länderübergreifend so geregelt, siehe auszugsweise z.B. in BadenWürttemberg: Art. 60 Abs. 1 LV BW; Bayern: Art. 74 Abs. 5 S. 1 BayLV; Hamburg: Art. 50 Abs. 3 S. 3 HmbLV; Nordrhein-Westfalen: Art. 68 Abs. 2 S. LV NW; Sachsen-Anhalt: Art. 81 Abs. 3 S. 1 LV LSA; Thüringen: Art. 82 Abs. 7 S. 2 ThürLV.

139 So explizit etwa in Baden-Württemberg, Art. 59 Abs. 1 LV BW, und Thüringen, Art. 81 Abs. 1 ThürLV, geregelt; als solches wird es auch bezeichnet in Hartmann, Volksgesetzgebung (Fn. 83), S. 110; Herrmann, Volksgesetzgebungsverfahren (Fn. 56), S. 117, spricht von der ,[Einbringung] der Vorlage in das Parlament“". 
immer noch zum Gesetzgeber berufenen Parlaments ${ }^{140}$, sie bedürfen also „Zwingend staatlicher Rezeption und Transformation“"141. Die Unterschriftenabgabe für das Volksbegehren ist nicht bereits selbst Ausübung von Hoheitsgewalt $^{142}$. Damit steht das Volksbegehren der staatlichen Willensbildung näher als die Volksinitiative, wenn sie auch noch nicht selbst unmittelbar Teil des Staatsgeschehens ist.

Im strikten Sinne sind demnach die Anforderungen des Demokratieprinzips nicht an die Finanzierung des Volksbegehrens anzulegen. Dies verkennt jedoch das unbedingte Erfordernis, vor einem Volksentscheid, der unter Ausübung von Hoheitsgewalt einzuordnen ist ${ }^{143}$, ein Volksbegehren anzustrengen. Könnte durch unüberwindliche und geradezu demokratiefeindliche Hürden der Volksentscheid mittelbar verhindert werden, indem unmittelbar das ,schutzlose“ Volksbegehren geregelt wird, würde das Demokratieprinzip im Bereich der direkten Demokratie praktisch leerlaufen. Es ist somit von Vorwirkungen des Demokratieprinzips auch auf das Volksbegehren auszugehen, wobei diese freilich im Vergleich zum Volksentscheid von geminderter Intensität sind. Dabei liegt in der Abgrenzung zur Volksinitiative keine willkürliche Zäsur vor ${ }^{144}$, weil im Gegensatz zur Volksinitiative im Anschluss an das Volksbegehren eine Entscheidung getroffen werden muss, nämlich entweder vom Parlament oder vom Volksgesetzgeber. Im Rahmen der Volksinitiative ist völlig unklar und keineswegs notwendig, dass sich eine solche Entscheidung anschließt.

Zur rechtlichen Bedeutung des Volksbegehrens kommen noch faktische Unterschiede zur Volksinitiative hinzu, die sich insbesondere in der verfahrensrechtlichen Ausgestaltung niederschlagen. Für das Volksbegehren ist das Erfordernis an Unterstützerstimmen markant erhöht, sei es im Rahmen einer freien Sammlung oder im Rahmen einer Amtseintragung. Die Konsequenzen dieser Steigerungen beschränken sich nicht allein auf den Sammelprozess

140 So auch Herrmann, Volksgesetzgebungsverfahren (Fn. 56), S. 116: „Erst die Legislativakte der Abstimmungsorgane setzen die plebiszitären Vorschläge als Hoheitsakte in die Willensbildungsprozesse des Rechtssubjektes Staat um“.

141 So die allgemeine Kennzeichnung von autonomer Entscheidungsgewalt bei Jestaedt, Demokratieprinzip (Fn. 40), S. 258, siehe dazu auch die Ausführungen unter III 4.

142 Anfechtbar insoweit Hartmann, Volksgesetzgebung (Fn. 83), S. 110, der sich auf OVG Münster, NVwZ 1998, S. 302 (302) bezieht. Dort wurde allerdings das Bürgerbegehren nach $\S 26$ Abs. 5 Nr. 9 NWGO als unzulässig abgelehnt, weil die beabsichtigte Aufforderung durch die Gemeinde, nicht aber das Bürgerbegehren selbst, gegen Grundrechte verstoße.

143 Dazu sogleich unter V.4.

144 So aber Hartmann, Volksgesetzgebung (Fn. 83), S. 110. 
selbst, der aber natürlich ebenfalls kostenintensiver wird. Unterschriftenquoren von $10 \%{ }^{145}$ oder gar $20 \%{ }^{146}$ verstärken diesen Effekt weiter. Das reale Kostenbedürfnis ist somit im Vergleich zur Volksinitiative gestiegen.

Die solchermaßen zu konstatierende gemischt staatlich-gesellschaftliche Struktur des Volksbegehrens unterliegt daher nicht in Gänze den strengen Maßstäben, wie sie aus dem Demokratieprinzip entwickelt worden sind. Es müsste demnach eine unerträgliche faktische Ungleichheit innerhalb der Wettbewerbssituation des Volksbegehrens geben. Dazu fehlen, wie oben bemerkt (V. 3.), jedoch noch empirische Untersuchungen. Hinzu kommt, dass das Volksbegehren nicht zwingend als antagonistischer Konflikt zwischen zwei konkurrierenden Regelungsmodellen aufgezogen wird, sondern auch als einseitige private Werbung um Unterstützerunterschriften durchgeführt werden kann ${ }^{147}$. Eine Kostenerstattungspflicht ist demnach für das Volksbegehren nicht anzunehmen. Es verbleibt aber im gesetzgeberischen Ermessen, eine Kostenerstattung einzuführen, hierzu bieten die doch immerhin teilweise vorhandenen Einwirkungen auf den staatlichen Willensbildungsprozess hinreichende Anknüpfungspunkte.

\section{Unterscheidung nach Erfolg}

Ebenfalls vor das prüfende Licht des Grundgesetzes zu ziehen ist die erfolgsabhängige Finanzierung etwa des Volksbegehrens oder des Volksentscheides. Die Unsicherheit, ob überhaupt Abstimmungskampfkosten erstattet werden, hat plausiblerweise Auswirkungen auf das Verhalten, insbesondere

145 Wie in Bayern, vgl. Art. 71 Abs. 2 BayLWahlG. Zusätzlich erschwert wird dies in Bayern dadurch, dass die Eintragungsfrist zwei Wochen beträgt, Art. 65 Abs. 3 S. 1 BayLWahlG.

146 So noch in Hessen, $\S 12$ Abs. 1 S. HessVoBegG, und bis zum 15. Mai 2013 im Saarland, Art. 99 Abs. 2 S. 3 LV SL a.F. - mittlerweile gilt dort eine Sieben-ProzentHürde, Art. 99 Abs. 2 S. 3 LV SL n.F. Diese Hürden trugen und tragen sicherlich dazu bei, dass in diesen Ländern ein Volksbegehren noch nie erfolgreich war.

147 Den „reaktiven Charakter" vieler Volksbegehren betont allerdings F. Decker, Volksgesetzgebung oder Volksveto? Überlegungen zur institutionellen Ausgestaltungen der Direktdemokratie in der Bundesrepublik, in: L.P. Feld/P.M. Huber/O. Jung/C. Welzel/F. Wittreck (Hrsg.), Jahrbuch für direkte Demokratie 2010, 2011, S. 37 (43 ff.); siehe dazu auch O. Jung, Die Reform der direkten Demokratie in Berlin 2006 (Teil II), in: L.P. Feld/P.M. Huber/O. Jung/C. Welzel/F. Wittreck (Hrsg.), Jahrbuch für direkte Demokratie 2012, 2013, S. 229 (273 ff.). Dies führt aber nicht schon dazu, dass die Konkurrenzsituation eines Volksbegehrens der eines Volksentscheides gleichstehen muss. 
die Mittelverwaltung, im Abstimmungskampf. Dies trifft vor allem beim Volksentscheid auf verfassungsrechtliche Bedenken. Ist nämlich dort die Mitwirkung Privater im Abstimmungskampf Teil eines staatlichen Geschehens, so kann nicht das Ergebnis der Abstimmung abschließendes Kriterium für die Finanzierung sein. Genauso wenig werden bei der Wahlkampffinanzierung nur die an einer Regierung beteiligten Parteien bedacht. Schließlich soll sich die demokratische Ausgestaltung gerade durch ihre Prozessgleichheit und Ergebnisoffenheit auszeichnen, nicht durch eine nachträgliche Subventionierung der Mehrheitsmeinung. Entscheidender Grund für die Finanzierungspflicht ist die unmittelbare Teilhabe der Akteure an der staatlichen Willensbildung. Diese Tätigkeit, die beim Volksentscheid in der Sache den Abstimmungskampf bedeutet, wird nun aber nicht im Nachhinein weniger oder mehr finanzierungswürdig. Beim Volksentscheid ist eine erfolgsabhängige Finanzierung daher unzulässig.

Bezüglich des Volksbegehrens hingegen kann insofern differenziert werden, als dieses direktdemokratische Instrument eine gesellschaftlichstaatliche Zwitterstellung einnimmt. Es ergibt sich danach keine unmittelbar ableitbare Kostenerstattungspflicht. Erst wenn das Volksbegehren erfolgreich ist, sich also das Parlament mit der Sache befassen muss, kann von einem staatlichen Vorgang gesprochen werden. Bleibt die Initiatorengruppe unter dem rechtlich vorgeschriebenen Unterschriftenquorum, kann noch von einem gesellschaftlichen Prozess gesprochen werden, wenn dieser auch Vorstufe zum staatlichen Geschehen ist. Eine Unterscheidung je nach Zustandekommen begegnet für das Volksbegehren daher keinen verfassungsrechtlichen Bedenken.

Gleiches gilt für die Volksinitiative. Dieses direktdemokratische Verfahren ist auch im Falle des Erfolgs noch dem gesellschaftlichen Bereich verhaftet, sodass keinesfalls von einer Finanzierungspflicht auszugehen ist und eine gesetzgeberische Regelung somit die Kostenerstattung nur im Erfolgsfall vorsehen kann.

\section{Kostenerstattung für direktdemokratische Instrumente de lege ferenda}

Nach dieser Bestandsaufnahme und Überprüfung der aktuellen Regelungssysteme gilt es nun, ausgehend von den verfassungsrechtlichen Geboten und den praktischen Erfahrungen, einen tragfähigen Vorschlag für die künftige Regelungskonzepte zu entwickeln. Angesichts der Ergebnisse dieser Untersuchung sind aus verfassungsrechtlichen Gründen in allen Bundesländern jedenfalls für den Volksentscheid Regelungen zur Kostenerstattung einzu- 
führen. Hinsichtlich der Höhe besteht dabei naturgemäß ein weiter Einschätzungsspielraum des Gesetzgebers, wobei faktische Beeinträchtigungen durch finanzielle Ungleichgewichte nach Möglichkeit vermieden werden müssen. Es ist mithin nicht notwendig, eine Kostendeckung in toto zu gewährleisten, solange verzerrende Wirkungen ausgeschlossen werden. Auf der gegenüberliegenden Seite des Regelungsspektrums kann erwogen werden, die Kosten für Volksinitiative und Volksbegehren zumindest rückwirkend $\mathrm{zu}$ ersetzen, indem ein entsprechend höherer Pauschalbetrag im Volksentscheid ausgezahlt wird. Auf diese Art kann der Gesetzgeber sowohl Volksinitiative als auch Volksbegehren als notwendige Vorstufen des Volksentscheides anerkennen, ohne zwingend für diese Instrumente eigene Finanzierungsmöglichkeiten vorzusehen ${ }^{148}$.

Anderes gilt hingegen für die Offenlegungs- und Rechenschaftspflichten $^{149}$. Für private Spenden erscheint dies nicht zwingend erforderlich und sollte in seiner Grundrechtsaffinität auch durchdacht sein. Im Regelfall sind die Interessengegensätze bei direktdemokratischen Verfahren klar umzeichnet, weshalb eine Offenlegung von Spenden aus Gründen der Information des Bürgers weniger dringlich erscheint. Hier dürfte aber ein Vergleich zu Wahlkampfspenden tragfähig sein, wonach ab einer gewissen Schwellensumme $^{150}$ Spender und Betrag offenzulegen sind ${ }^{151}$. Im Übrigen ist aber zu berücksichtigen - worauf auch der US Supreme Court in seiner Rechtsprechung verweist ${ }^{152}$-, dass die Korruptionsgefahr bei Sachplebisziten plausiblerweise geringer einzuschätzen ist als bei personalisierten Wahlen. Anderes gilt für die Rechenschaftspflicht: Wer öffentliche Gelder einsetzt, muss deren zweckgetreue Verwendung nachweisen, zumindest in Höhe der Kostenerstattung ist daher die Ausgabenseite kundzutun ${ }^{153}$.

148 In rechtspolitischer Hinsicht hält Jung, Regeln (Fn. 7), S. 52, die Kostenerstattung für das Volksbegehren ebenfalls für zweckmäßig, dies allerdings ohne normative Anknüpfung und nur mit dem Hinweis auf die „Politische Kultur der Bundesrepublik".

149 Ausführlich und durchdacht zu möglichen rechtspolitischen Entwürfen für die Schweiz Caroni, Herausforderung (Fn. 9), S. 87 ff.

150 Für natürliche Personen derzeit $10.000 €, \S 25$ Abs. $3 \mathrm{~S} .1$ PartG.

151 Mit Art. 21 Abs. 1 S. 4 GG im verfassungsrechtlichen Hintergrund hielt das Bundesverfassungsgericht eine Offenlegung erst ab 40.000 DM sogar für $\mathrm{zu}$ hoch, BVerfGE 85, 264 (319 ff.).

152 Zum ersten Mal 1978 benannt in First National Bank of Boston v. Bellotti - 435 U.S. 765 (790).

153 Die Publizität als Folgerung aus dem Demokratieprinzip betont bereits Schmitt, Verfassungslehre (Fn. 41), S. 208 f.; zur demokratischen Publizität sowie deren Bedeu- 
Es bleibt festzuhalten: Aus verfassungsrechtlichen Gründen ist jeder Landesgesetzgeber gehalten, eine Kostenerstattung für Abstimmungskampfkosten zumindest für den Volksentscheid vorzusehen. Diese kann nicht vom Ergebnis der Abstimmung abhängen, muss aber gleichzeitig nicht den gesamten Mittelaufwand der Akteure abdecken. Für das Volksbegehren ist eine staatliche Finanzierung dagegen nicht zwingend, ebensowenig für die Volksinitiative.

Folgt der Gesetzgeber diesem Regelungsmodell - auch wenn ihm in concreto ein weiter Gestaltungsspielraum zukommt -, so können die Verfahren der direkten Demokratie ein bedeutender (oder: noch bedeutenderer) Bestandteil des politischen Gefüges der Bundesländer werden. Dabei ist eine Kostenerstattung nicht Allheilmittel für real oder vermeintlich vorhandene Politikmüdigkeit. Aber abgesehen von der symbolischen Aufwertung der Verfahren der unmittelbaren Demokratie durch staatliche Gelder wird damit eine weitere faktisch abschreckende Barriere abgebaut, die bürgerliches Engagement bislang hinderte. Wenn durch die unmittelbare Demokratie Ausübung von Staatsgewalt direkt dem Volk übertragen werden soll, dann darf diese Entscheidung nicht durch faktisch erhebliche Einflussnahme einiger weniger konterkariert werden. In diesem Sinne kann die Kostenerstattung einen Beitrag zur Beantwortung einer Frage stellen, die schon Bertolt Brecht umgetrieben hat: „Die Staatsgewalt geht vom Volke aus. - Aber wo geht sie hin?"،154

tung in der Parteienfinanzierung Reichel, Offenheitsprinzip (Fn. 64), S. 110 ff., 126 ff.

154 So kommentierte Brecht Art. 1 Abs. 2 WRV in seinem Gedicht Paragraph 1: $B$. Brecht, Die Gedichte in einem Band, hrsg. von E. Hauptmann, 2003, S. 378. 
